ARTICLE

\title{
Palmitoylation targets the calcineurin phosphatase to the phosphatidylinositol 4-kinase complex at the plasma membrane
}

Idil Ulengin-Talkish (1) 1, Matthew A. H. Parson (10 2 ${ }^{2}$, Meredith L. Jenkins², Jagoree Roy ${ }^{1}$, Alexis Z. L. Shih (10 3,9, Nicole St-Denis ${ }^{4,10}$, Gergo Gulyas (10 ${ }^{5}$, Tamas Balla ${ }^{5}$, Anne-Claude Gingras (1) 4,6, Péter Várnai (10) ${ }^{7}$,

Elizabeth Conibear ${ }^{3}$, John E. Burke (10) ${ }^{2,8} \&$ Martha S. Cyert (10 ${ }^{1 \times}$

Calcineurin, the conserved protein phosphatase and target of immunosuppressants, is a critical mediator of $\mathrm{Ca}^{2+}$ signaling. Here, to discover calcineurin-regulated processes we examined an understudied isoform, CNA $\beta 1$. We show that unlike canonical cytosolic calcineurin, CNA $\beta 1$ localizes to the plasma membrane and Golgi due to palmitoylation of its divergent C-terminal tail, which is reversed by the ABHD17A depalmitoylase. Palmitoylation targets $C N A \beta 1$ to a distinct set of membrane-associated interactors including the phosphatidylinositol 4-kinase (PI4KA) complex containing EFR3B, PI4KA, TTC7B and FAM126A. Hydrogen-deuterium exchange reveals multiple calcineurin-PI4KA complex contacts, including a calcineurin-binding peptide motif in the disordered tail of FAM126A, which we establish as a calcineurin substrate. Calcineurin inhibitors decrease PI4P production during Gq-coupled GPCR signaling, suggesting that calcineurin dephosphorylates and promotes PI4KA complex activity. In sum, this work discovers a calcineurin-regulated signaling pathway which highlights the PI4KA complex as a regulatory target and reveals that dynamic palmitoylation confers unique localization, substrate specificity and regulation to CNAB1.

\footnotetext{
${ }^{1}$ Department of Biology, Stanford University, Stanford, CA, USA. ${ }^{2}$ Department of Biochemistry and Microbiology, University of Victoria, Victoria, BC, Canada. ${ }^{3}$ Department of Medical Genetics, University of British Columbia, Vancouver, Canada. ${ }^{4}$ Lunenfeld-Tanenbaum Research Institute at Mount Sinai Hospital, University of Toronto, Toronto, Canada. ${ }^{5}$ Section on Molecular Signal Transduction, National Institute of Child Health and Human Development, National Institutes of Health, Bethesda, MD, USA. ${ }^{6}$ Department of Molecular Genetics, University of Toronto, Toronto, ON, Canada. 7 Department of Physiology, Faculty of Medicine, Semmelweis University, Budapest, Hungary. ${ }^{8}$ Department of Biochemistry, The University of British Columbia, Vancouver, BC, Canada. ${ }^{9}$ Present address: Max-Delbrück Center for Molecular Medicine, Berlin, Germany. ${ }^{10}$ Present address: High-Fidelity Science Communications, Summerside, PE,

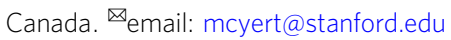


C ells respond to changes in their environment via signaling pathways, including those regulated by calcium ions $\left(\mathrm{Ca}^{2+}\right)$. Dynamic changes in the intracellular $\mathrm{Ca}^{2+}$ concentration provide specific temporal and spatial cues that direct a myriad of physiological responses. Hence, elucidating mechanisms that initiate $\mathrm{Ca}^{2+}$ signaling and identifying downstream $\mathrm{Ca}^{2+}$ sensing-effectors are critical for understanding cellular regulation in both healthy and diseased cells.

Calcineurin $(\mathrm{CN})$, also known as $\mathrm{PP} 2 \mathrm{~B}$ or PPP3, is the conserved $\mathrm{Ca}^{2+} /$ calmodulin $(\mathrm{CaM})$-activated serine/threonine protein phosphatase, that transduces $\mathrm{Ca}^{2+}$ signals to regulate a widearray of physiological processes. In humans, $\mathrm{CN}$ is ubiquitously expressed and has well-established roles in the cardiovascular, nervous, and immune systems ${ }^{1-3}$. Because $\mathrm{CN}$ dephosphorylates Nuclear Factor of Activated T-cells (NFAT) transcription factors to activate the adaptive immune response ${ }^{4}, \mathrm{CN}$ inhibitors FK506 (Tacrolimus), and cyclosporin A (CsA) are in wide clinical use as immunosuppressants ${ }^{5}$. However, by inhibiting $\mathrm{CN}$ in nonimmune tissues, these drugs also provoke a variety of unwanted effects, underscoring the need to comprehensively map $\mathrm{CN}$ signaling throughout the body. Recently, systematic discovery of $\mathrm{CN}$ targets revealed that many $\mathrm{CN}$-regulated pathways are yet to be elucidated $^{6,7}$. Here, we uncover aspects of $\mathrm{CN}$ signaling by focusing on an understudied isoform, CNA $\beta 1$.

Calcineurin is an obligate heterodimer of catalytic (CNA) and regulatory $(\mathrm{CNB})$ subunits. In mammals, three isoforms of CNA $(\alpha, \beta$ and $\gamma$ ) are encoded by separate genes with tissue-specific expression. These isoforms display a similar architecture containing a catalytic domain, binding sites for $\mathrm{CNB}$ and $\mathrm{CaM}$, and a C-terminal autoinhibitory domain (AID) which blocks phosphatase activity under basal conditions. Under elevated $\mathrm{Ca}^{2+}$ conditions, $\mathrm{Ca}^{2+}$ and $\mathrm{Ca}^{2+} / \mathrm{CaM}$ bind to $\mathrm{CNB}$ and $\mathrm{CNA}$, respectively, to disrupt $\mathrm{AID}$ binding to the catalytic site ${ }^{8,9}$. This activation mechanism is conserved across all $\mathrm{CN}$ isoforms in animals and fungi, with the only known exception being a transcript variant of the CNA $\beta$ gene, termed $\mathrm{CNA} \beta 1^{10-13}$.

Alternative $3^{\prime}$ end processing of the $P P P 3 C B$ mRNA gives rise to two CNA $\beta$ isoforms, $\mathrm{CNA} \beta 2$ with canonical architecture, and the non-canonical CNA $\beta 1^{10,11}$ (Fig. 1a). CNA $\beta 1$ is conserved in vertebrates (Fig. 1b) and broadly expressed in human tissues at a low level, alongside the canonical $\mathrm{CN}$ isoforms ${ }^{11,12}$. CNA $\beta 1$ and $\mathrm{CNA} \beta 2$ sequences are identical through the CaM-binding domain, but exclusion of two terminal exons and subsequent translation of intronic sequences results in a divergent hydrophobic C-terminus for CNA $\beta 1$ that lacks the AID, but contains a distinct autoinhibitory sequence, ${ }^{462} \mathrm{LAVP}^{465}$, which impedes substrate binding ${ }^{12}$. CN recognizes two short, degenerate peptide motifs, "PxIxIT" and "LxVP", in the disordered regions of its substrates ${ }^{13}$. LxVP motifs bind to a region at the $\mathrm{CNA} / \mathrm{CNB}$ interface that is accessible only after $\mathrm{Ca}^{2+} / \mathrm{CaM}$ binding ${ }^{13-15}$ and is blocked by FK506 and CsA showing that LxVP recognition is essential for dephosphorylation ${ }^{15}$. Notably, the unique LxVP-mediated autoinhibition displayed by $\mathrm{CNA} \beta 1$ is only partially relieved by $\mathrm{Ca}^{2+} / \mathrm{CaM}$ and limits maximal activity in vitro when compared to $\mathrm{CNA} \beta 2^{12}$. However, regulation of $\mathrm{CNA} \beta 1$ in vivo remains to be investigated.

The current $\mathrm{CN}$ literature is focused on canonical isoforms, however the few published studies about CNA $\beta 1$ reveal its unique physiological roles: In mouse cardiomyocytes CNA $\beta 1$ overexpression is cardio-protective following myocardial infarction, in contrast to pro-hypertropic $\mathrm{CNA} \beta 2^{16-18}$. Furthermore, mice specifically lacking $\mathrm{CNA} \beta 1$ are viable, but exhibit cardiac hypertrophy and metabolic alterations ${ }^{18}$. CNA $\beta 1$ also regulates mouse embryonic stem cell differentiation and activates mTORC2/AKT signaling through an undetermined mechanism that may be independent of its catalytic activity ${ }^{11,16,19}$. Additionally, unlike $\mathrm{CNA} \beta 2, \mathrm{CNA} \beta 1$ does not dephosphorylate
NFAT $^{16}$, and its direct substrates are yet to be identified. Thus, elucidation of these targets promises to reveal unknown aspects of $\mathrm{Ca}^{2+}$ and $\mathrm{CN}$ signaling.

Some of the best-characterized $\mathrm{Ca}^{2+}$ signaling pathways are initiated by ligand binding to Gq-protein coupled receptors (GPCR), causing phospholipase $\mathrm{C}$ (PLC) to hydrolyze phosphatidylinositol 4,5-biphosphate $\left(\mathrm{PI}(4,5) \mathrm{P}_{2}\right.$ or $\left.\mathrm{PIP}_{2}\right)$ into diacylglycerol $(\mathrm{DAG})$ and inositol triphosphate $\left(\mathrm{IP}_{3}\right)$, which activate protein kinase $\mathrm{C}(\mathrm{PKC})$ and intracellular $\mathrm{Ca}^{2+}$ release, respectively ${ }^{20}$. Therefore, sustained $\mathrm{Ca}^{2+}$ signaling through GPCRs requires continued phosphorylation of plasma membrane (PM) phosphatidylinositol (PI) to generate phosphatidylinositol 4-phosphate (PI4P), the precursor of $\mathrm{PI}(4,5) \mathrm{P}_{2}$. Indeed, real-time monitoring of $\mathrm{PM}$ phospholipid levels during GPCR signaling reveals that, concomitant with $\mathrm{PI}(4,5) \mathrm{P}_{2}$ depletion, phosphatidylinositol 4-kinase IIIa (PI4KA) is activated to increase PI4P synthesis ${ }^{21,22}$.

PI4KA is recruited to the PM by at least two accessory proteins, EFR3A/B and TTC7A/B, which are conserved from yeast to mammals ${ }^{23-26}$. EFR3 is stably associated with the PM via palmitoylation, and anchors the complex to the membrane, while TTC7 (Ypp1 in yeast) binds to both EFR3 and PI4KA (Stt4 in yeast) and acts as the shuttle ${ }^{27}$. A third protein, either FAM126A (Hyccin) or FAM126B, is an essential regulatory component, present only in higher eukaryotes, that stabilizes the TTC7PI4KA interaction in the cytosol, and enhances PI4KA recruitment to the $\mathrm{PM}^{24}$. PI4KA/TTC7/FAM126A heterotrimers form a dimer, and this super-assembly likely stabilizes and orients the PI4KA active site toward the membrane to promote its activity ${ }^{28}$. Furthermore, the disordered C-terminus of FAM126A is not visible in existing structures, and modulates the PI4KA catalytic activity in vitro through an unknown mechanism ${ }^{29}$. This intricate structure suggests that both the assembly and activity of the PI4KA complex are tightly regulated. In yeast, PI4KA recruitment to the PM is regulated by phosphorylation ${ }^{26}$. However, regulation of PI4KA complex assembly and/or activity in mammals remains to be elucidated.

This work discovers $\mathrm{CN}$ functions by focusing on the CNA $\beta 1 /$ $\mathrm{CNB}$ isozyme. We demonstrate that unlike the cytosolic, canonical CNA $\beta 2$, CNA $\beta 1$ localizes to cellular membranes, primarily the PM and Golgi apparatus, via palmitoylation of two conserved cysteines within its unique C-terminus. The ABHD17A thioesterase depalmitoylates CNA $\beta 1$ causing its redistribution and suggesting that this dynamic palmitoylation regulates CNA $\beta 1$ signaling in vivo. To identify potential substrates of $\mathrm{CNA} \beta 1$, we carried out affinity purification coupled to mass spectrometry (AP-MS) which revealed CNA $\beta 1$-specific interactors to be largely membrane-associated, and unexpectedly identified all four members of the PI4KA complex. Using in vivo and in vitro analyses, including hydrogen deuterium exchange mass spectrometry (HDX-MS), we identified multiple sites of CN-PI4KA complex association, including direct interaction with a short linear motif, PSISIT, within the unstructured C-terminal tail of FAM126A. Our studies establish FAM126A as a CN substrate that preferentially interacts with $\mathrm{CNA} \beta 1$ at the PM. Finally, we uncover a role for $\mathrm{CN}$ in PI4P production at the PM by PI4KA during signaling from the type- 3 muscarinic receptor. In total, this work discovers a CN-regulated signaling pathway that highlights the PI4KA complex as a regulatory target and demonstrates that palmitoylation dictates substrate specificity of the non-canonical CNA $\beta 1$ isoform.

\section{Results}

CNA $\beta 1$ localizes to the plasma membrane, Golgi apparatus, and intracellular vesicles. We sought to investigate the unique functions of CNA $\beta 1$ by characterizing it's in vivo properties. 
a

\begin{tabular}{|c|c|c|c|c|c|}
\hline \multirow[b]{2}{*}{ CNAß2 } & & & & \multicolumn{2}{|c|}{ 'Canonical C-terminus } \\
\hline & Catalytic Domain & CNB & $\mathrm{CaM}$ & & AID \\
\hline & & & & \multicolumn{2}{|c|}{ Alternative C-terminus } \\
\hline CNA $\beta 1$ & Catalytic Domain & CNB & CaM & LAVP C C & \\
\hline
\end{tabular}

b

H.sapiens GNDVMCLAVPQMDWGTPHSFANNSHNACREFLLFFSSCLSS P.troglodytes GNDVMC LAVP QMDWGT PHSF ANN SHNACREFLL LF SSCLSS S.scufa GNDVMC LAVP QMDWGTPHSFANNTHNACREFFLLFSSCLSS D.rotundus GNDVMC LAVPQMDWGT PHSFANNTHNACREFLLLFSSCLSS M.musculus GNDVMC LAVPQMDWGTTHSFANNTHNACRELLLLFSSCLSS C. milii GNDALFLPVPQMEWGTSHCFNGT TRNACPDFLMLFSSCLSG X.tropicalis GNDT - CLAVPRWT GAH I I PFPNST PNACWEF LMLFRSCL SG

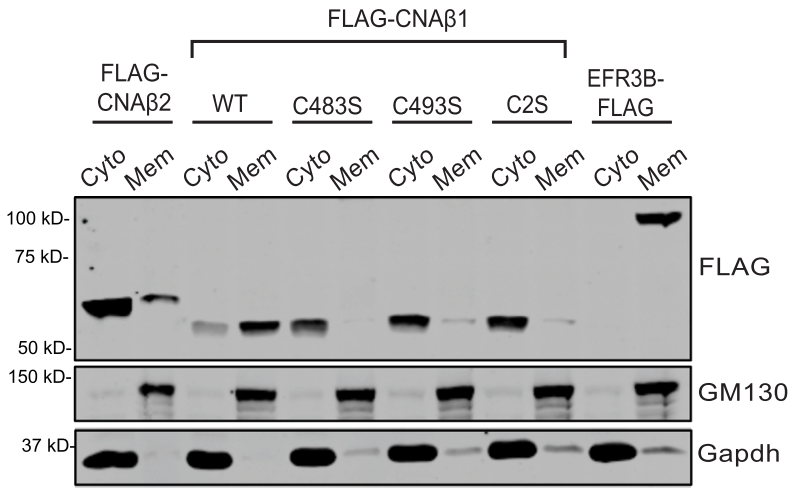

e
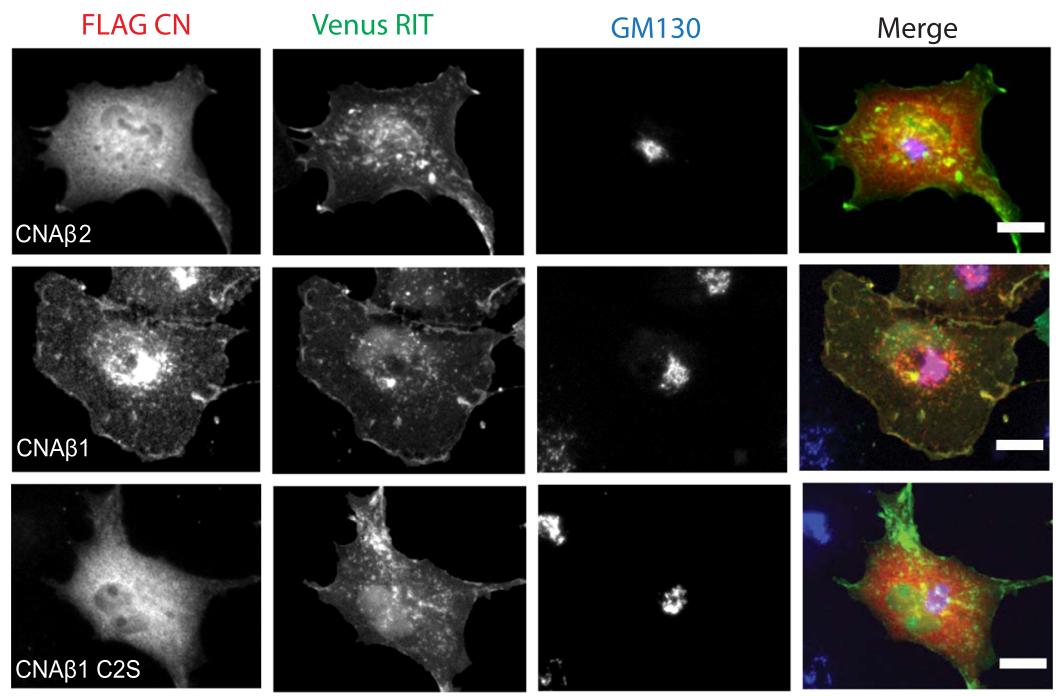

$\mathbf{g}$
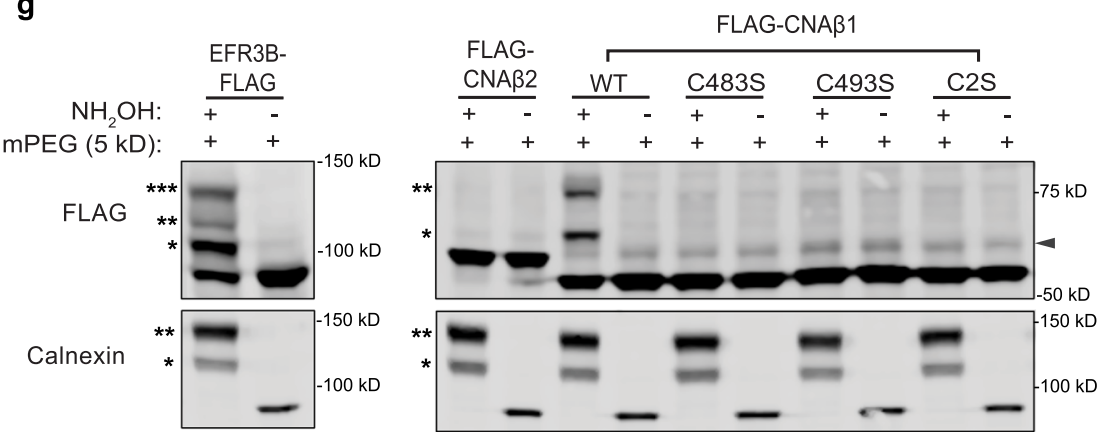
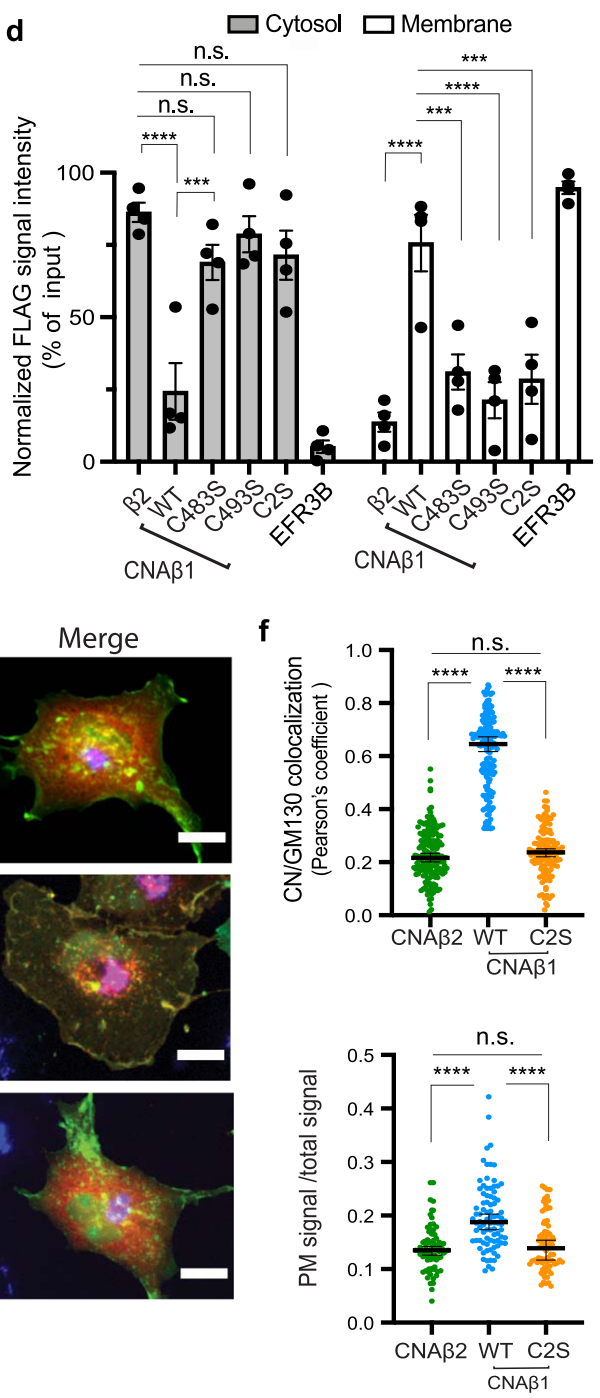

First, we analyzed the intracellular distribution of CNA $\beta 1$, which was previously found to be Golgi-associated in mouse embryonic stem cells ${ }^{19}$. Subcellular fractionation of COS-7 cells expressing FLAG-tagged CNA $\beta 1$ or CNA $\beta 2$, confirmed that CNA $\beta 1$ was highly enriched in membranes while CNA $\beta 2$ was primarily cytosolic (Fig. 1c, d). Furthermore, indirect immunofluorescence of these cells revealed that CNA $\beta 1$ localized to the PM, where it overlapped significantly with a co-expressed PM marker (Venus-RIT) ${ }^{30}$, the Golgi apparatus, where it co-localized with GM130, and to intracellular vesicles. By contrast, CNA $\beta 2$ was predominantly cytosolic with minimal co-localization with either membrane marker (Fig. 1e, f). Similar distributions were observed in HeLa cells (Supplementary Fig. 1a). 
Fig. 1 CNA $\beta 1$ localizes to intracellular membranes via palmitoylation at two conserved cysteines unique to its C-terminal tail. a Schematic of $C N A \beta$ isoforms. CNB and calmodulin binding domains (CNB and CaM); autoinhibitory domain, (AID, blue); LAVP autoinhibitory sequence (green); palmitoylated cysteines (red). b CNAB1 C-terminal (a.a. 456-496) sequence alignment; autoinhibitory LAVP (green) and palmitoylated cysteines (red, C483 and C493), are boxed. c Representative immunoblot demonstrating subcellular fractionation of COS-7 cells transfected with FLAG-CNA 32 , -CNA $\beta 1$ (WT or cysteine mutants), or EFR3B-FLAG using anti-FLAG. GM130 and Gapdh define membrane and cytosol fractions, respectively ( $n=4$ independent experiments). d Quantification of four independent experiments as in c. Data show the mean \pm SEM. n.s. not significant, ${ }^{\star \star *} p<0.001$ : cytosol, WT vs C483S $p=0.0004$; membrane, WT vs C483S $p=0.0004$, WT vs C2S $p=0.0002$; ${ }^{\star \star \star \star} p<0.0001$ using two-way ANOVA with Holm-Sidaks multiple comparison tests. e Representative images of COS-7 cells expressing FLAG-tagged CNA $\beta 2$, CNA $\beta 1$ or CNA $\beta 1^{\mathrm{C} 2 \mathrm{~S}}$ with Venus-Rit (PM, green). Fixed cells immunostained with anti-FLAG (red) and anti-GM130 (blue). Scale bar $=15 \mu \mathrm{m}$. f Top graph: Co-localization of FLAG signal (as in e) with Golgi marker, GM130. Data show median of Pearson's coefficients with $95 \%$ confidence intervals ( $\mathrm{Cl}$ ), from $\geq 100$ cells analyzed in three independent experiments (see Statistical Analysis). n.s not significant; ${ }^{\star \star \star \star} p<0.0001$, using one-way ANOVA followed by Kruskal-Wallis test. Bottom graph: PM localization quantified as anti-FLAG signal intensity at cell periphery (defined in Supplementary Fig. 1d) over total cell intensity (see Methods section). Data show median with $95 \% \mathrm{Cl}$ from $\geq 70$ cells imaged in three independent experiments. ${ }^{\star \star \star} p<0.0001$ using one-way ANOVA followed by Kruskal-Wallis test. $\mathbf{g}$ Representative immunoblot of AcylPEG exchange performed on lysates of COS-7 cells transfected with FLAG-CNAB2, FLAG -CNA 1 (WT or cysteine mutants) or EFR3B-FLAG. The number of PEGylation (reflecting S-palmitoylation) events are indicated by asterisks. Arrowhead indicates non-specific antibody band. $n \geq 3$ independent experiments for all constructs (see Statistical Analysis).

CNA $\beta 1$ is palmitoylated at two conserved cysteines unique to its C-terminal tail. S-Palmitoylation, the reversible addition of a 16-carbon fatty acid chain to cysteine residues via a thioester linkage, allows proteins lacking a transmembrane domain to associate with cellular membranes ${ }^{31}$. We noted that the C-terminus of CNA $\beta 1$ contains two highly conserved cysteines: C483, which is contained within a previously defined "Golgi localization domain"19,32 and C493, which is predicted as a highconfidence S-palmitoylation site ${ }^{33}$ (Fig. 1a, b). Thus, we investigated possible palmitoylation of CNA $\beta 1$ using acyl resin-assisted capture (Acyl-RAC), during which the thioester linkage in palmitoylated cysteines is cleaved with hydroxylamine $\left(\mathrm{NH}_{2} \mathrm{OH}\right)$ to allow protein binding to thiopropyl-sepharose beads ${ }^{34}$. Acyl-RAC analysis of FLAG-CNA $\beta 2$, FLAG-CNA $\beta 1$, or EFR3B-FLAG expressed in COS-7 cells revealed the presence of S-palmitoylated cysteines in our positive control, EFR3B and in $\mathrm{CNA} \beta 1$, but not CNA $\beta 2$ (Supplementary Fig. 1b). Furthermore, $\mathrm{CNA} \beta 1$ mutants containing either single or double serine substitutions at $\mathrm{C} 483$ and/or $\mathrm{C} 493$, from here on referred to as $\mathrm{CNA} \beta 1^{\mathrm{C} 483 \mathrm{~S}}, \mathrm{CNA} \beta 1^{\mathrm{C} 493 \mathrm{~S}}$, and $\mathrm{CNA} \beta 1^{\mathrm{C} 2 \mathrm{~S}}$ respectively, were not captured by Acyl-RAC, suggesting that at least one of these residues is palmitoylated (Supplementary Fig. 1b). To determine the stoichiometry of CNA $\beta 1$ palmitoylation, we used acyl-PEG exchange (APE) in which the palmitate groups on modified cysteines are removed by hydroxylamine and replaced with a mass-tag (mPEG) that causes a $5 \mathrm{kDa}$ mass-shift for each acylated cysteine ${ }^{35}$. Mass-tag conversion may be incomplete during APE; thus, this method accurately reports S-acylation states, but not the fraction of protein in the sample that is modified ${ }^{36}$. Our positive controls were EFR3B, which contains three palmitoylated cysteines, and the endogenous ER chaperone, calnexin, which is dually palmitoylated ${ }^{27,37}$. As expected, EFR3B-FLAG and calnexin showed three and two mass-shifted bands, respectively, while cytosolic FLAG-CNA $\beta 2$ showed no shifts (Fig. 1g). Interestingly, FLAG-CNA $\beta 1$ displayed two mass-shifted forms indicating two sites of palmitoylation, but mutants, $\mathrm{CNA} \beta 1^{\mathrm{C} 483 \mathrm{~S}}$, $\mathrm{CNA} \beta 1^{\mathrm{C} 493 \mathrm{~S}}$ or $\mathrm{CNA} \beta 1^{\mathrm{C} 2 \mathrm{~S}}$, displayed no electrophoretic shifts. Thus, both CNA $\beta 1$ cysteines are apparently required for stable palmitoylation suggesting that palmitoylation is cooperative, as described for calnexin ${ }^{37}$.

Palmitoylation is required for CNA $\beta 1$ membrane association. To determine if palmitoylation mediates CNA $\beta 1$ membrane association, we first metabolically labeled COS-7 cells with the palmitate analog, 17-octadecynoic acid (17-ODYA) and showed that upon subcellular fractionation, the majority of the 17ODYA-labeled CNA $\beta 1$ was in the membrane fraction
(Supplementary Fig. 1c). Next, we analyzed the fractionation of palmitoylation-defective mutants $\mathrm{CNA} \beta 1^{\mathrm{C} 483 \mathrm{~S}}, \mathrm{CNA} \beta 1^{\mathrm{C} 493 \mathrm{~S}}$, and $\mathrm{CNA} \beta 1^{\mathrm{C} 2 \mathrm{~S}}$ which, in contrast to wildtype CNA $\beta 1$, were predominantly enriched in the cytosolic fractions (Fig. 1c, d). Finally, we examined each mutant using indirect immunofluorescence. As expected, FLAG-CNA $\beta 1^{\mathrm{C} 493 \mathrm{~S}}$ and FLAG-CNA $\beta 1^{\mathrm{C} 2 \mathrm{~S}}$ mutants were cytosolic and did not co-localize with either PM or Golgi membrane markers (Venus RIT and GM130, respectively) (Fig. 1e, f and Supplementary Fig. 1e, f). Interestingly, although FLAG-CNA $\beta 1^{\mathrm{C} 483 \mathrm{~S}}$ was predominantly cytosolic, a minority of cells exhibited weak Golgi and PM localization (Supplementary Fig. 1e (top panel, red and green boxes), f), suggesting that this mutant might be palmitoylated at a low level that is insufficient for stable membrane association. Thus, palmitoylation of Cys493 may serve as the priming site for Cys483 palmitoylation. In sum, these analyses show that both Cys483 and Cys493 are palmitoylated and that dual palmitoylation is required for the stable association of CNA $\beta 1$ with membranes, particularly with the PM. Therefore, the unique lipidated C-terminal tail of CNA $\beta 1$ confers distinct localization to this isoform.

CNA $\beta 1$ palmitoylation is dynamically regulated. Protein palmitoylation is reversed by depalmitoylases, and some proteins, including RAS GTPases, undergo rapid palmitate turnover during their lifespan, which regulates their localization and function 38,39 . To determine palmitoylation dynamics for CNA $\beta 1$, we monitored S-acylation while simultaneously controlling for protein turnover using a dual label pulse-chase experiment. Cells were labelled with both palmitate (17-ODYA) and methionine (L-azidohomoalanine or L-AHA) analogs, which were later visualized via click chemistry using AF647-azide and AF488-alkyne, respectively ${ }^{39-41}$. COS-7 cells expressing FLAG-CNA $\beta 1$ were briefly labelled with both analogs and then chased with media lacking the analogs but containing either Palmostatin B (Palm B), a pan inhibitor of depalmitoylases, or vehicle (DMSO) (Fig. 2a). FLAG-CNA $\beta 1$ was then immunopurified and the level of 17ODYA incorporated into the pool of L-AHA labelled CNA $\beta 1$ (17-ODYA/L-AHA) was determined as a function of time. Palm $B$ treatment caused a marked increase in the ratio of 17-ODYA/LAHA labelled FLAG-CNA $\beta 1$ over time relative to control cells where palmitate turnover occurred (Fig. 2b, c). Thus, palmitoylation of CNA $\beta 1$ is dynamic and is actively reversed by depalmitoylases in vivo.

In mammals, two classes of thioesterases are responsible for protein depalmitoylation. The soluble, acyl protein thioesterases (APT1, APT2) and the membrane-associated $\alpha / \beta$ hydrolase domain proteins (ABHD17s) display distinct substrate 
a

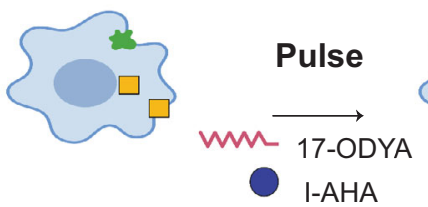

b
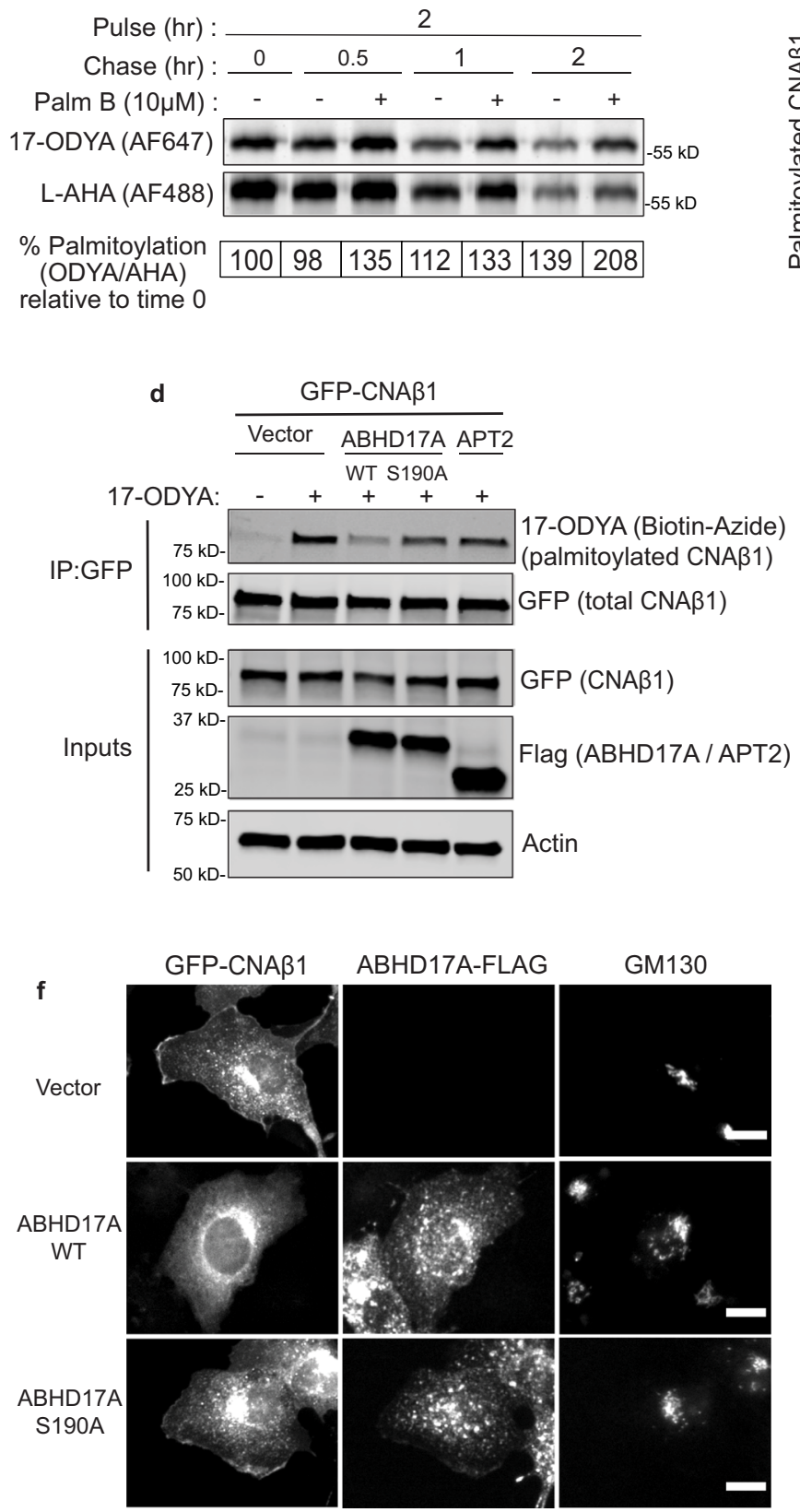

Chase

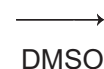

Palm B

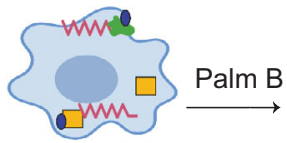

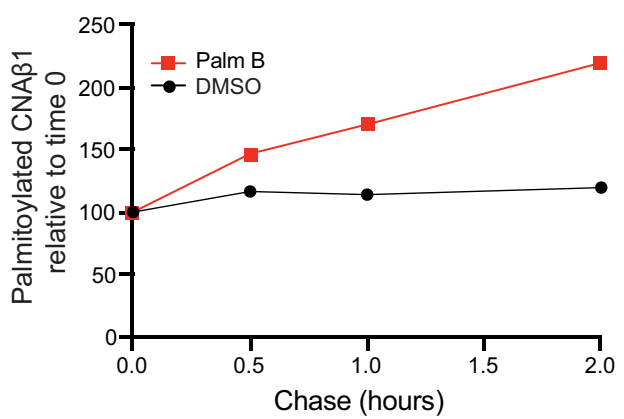
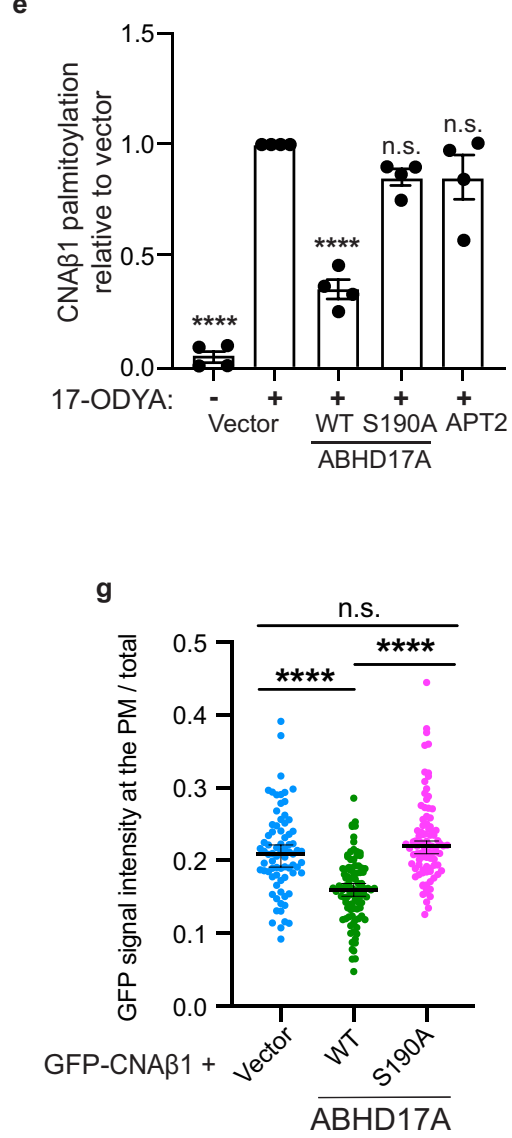

specificities likely due to their differential subcellular distribution $^{39,40}$. Among the ABHD17 family, we focused on the well-characterized ABHD17A, which localizes to the PM, Golgi, and endosomes ${ }^{39}$. To identify which of these thioesterases act on CNA $\beta 1$, we examined 17-ODYA labeling of GFP-CNA $\beta 1$ co-expressed in COS-7 cells together with a vector, ABHD17AFLAG (WT or the catalytically impaired mutant $S 190 \mathrm{~A}^{39}$ ),
FLAG-APT2, or mCherry-APT1. Overexpression of ABHD17A WT, but not S190A, dramatically decreased the 17-ODYA labeling of GFP-CNA 1 (Fig. 2d, e), and resulted in redistribution of GFP-CNA $\beta 1$ from the PM to the cytosol and Golgi (Fig. 2f, g), suggesting that ABHD17A depalmitoylates CNA $\beta 1$. Furthermore, APE analysis showed that, compared to vector and ABHD17A (S190A), expression of ABHD17A WT significantly reduced 


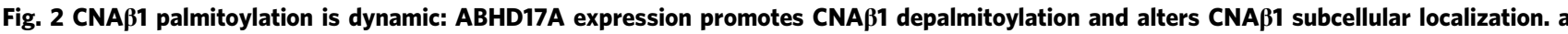

Schematic diagram of the pulse-chase experiment using analogs of palmitate (17-ODYA) and methionine (L-AHA) coupled to CLICK chemistry used in this study. $\mathbf{b}$ Pulse-chase analysis of palmitate turnover on FLAG-CNA $\beta 1$ by dual-click chemistry as described in $\mathbf{a}$ in the presence of DMSO or pandepalmitoylase inhibitor Palm B. Representative in-gel fluorescence scans showing dual detection of 17-ODYA and L-AHA using Alexa Fluor 647 and Alexa Fluor 488, respectively. c Time course of FLAG-CNA 1 depalmitoylation in DMSO- and Palm B-treated cells after normalizing 17-ODYA to L-AHA signals at each chase time. Data shown are mean of each time point from two independent experiments. d Analysis of GFP-CNA 1 palmitoylation co-expressed with vector, ABHD17A-FLAG (WT or S190A) or FLAG-APT2, using metabolic labeling with 17-ODYA. Representative immunoblot illustrates total CNA 1 using anti-GFP and 17-ODYA detected using streptavidin following CLICK chemistry with Biotin-Azide. Anti-FLAG shows amount of ABHD17A and APT2 expression ( $n=4$ independent experiments). e GFP-CNA $\beta 1$ palmitoylation (as in $\mathbf{d}$ ) is quantified by the streptavidin signal (17-ODYA)/total protein signal (GFP) and normalized to vector control. Data are mean \pm SEM. ( $n=4$ independent experiments) n.s. not significant, ${ }^{\star \star \star \star} p<0.0001$ using one-way ANOVA with Dunnett's multiple comparison tests. $\mathbf{f}$ Representative images of fixed, COS-7 cells co-expressing GFP-CNA $\beta 1$ with vector, ABHD17A-FLAG (WT or S190A) immunostained with anti-FLAG and anti-GM130 (Golgi). Scale bar $=15 \mu \mathrm{m}$. $\mathbf{g}$ Images (as in f) quantified as GFP signal at the PM relative to total GFP signal intensity; data show median with $95 \%$ confidence intervals. $\geq 75$ cells quantified per condition from four independent experiments. (see Statistical Analysis). n.s. not significant, ${ }^{\star \star \star \star} p<0.0001$ using one-way ANOVA followed by Kruskal-Wallis test.

palmitoylation of $\mathrm{CNA} \beta 1$, especially the dually palmitoylated form that is required for stable PM association (Supplementary Fig. 2a). This is consistent with CNA $\beta 1$ localization to the Golgi in these cells. In contrast, overexpression of APT2 (Fig. 2d, e) or APT1 (Supplementary Fig. 2b, c) did not alter palmitoylation of CNA $\beta 1$. Together, these data reveal that CNA $\beta 1$ is dynamically palmitoylated, which may regulate its localization in vivo. In sum, we show distinct cellular properties for CNA $\beta 1$, compared to canonical $\mathrm{CN}$ isoforms, which led us to search for CNA $\beta 1$ specific substrates and functions.

Affinity purification and mass spectrometry identifies CNA $\beta 1$ specific interactors. Previous studies report that, unlike canonical $\mathrm{CN}$ isoforms, CNA $\beta 1$ does not activate or interact with $\mathrm{NFAT}^{16}$. Indeed, when FLAG-NFATC1 was co-expressed in HEK293 FlpIn T-REx cell lines that inducibly express GFP, GFP-CNAa, GFP$\mathrm{CNA} \beta 2$, or GFP-CNA $\beta 1$, immunoprecipitation of GFP-CNs confirmed that NFATC1 co-purifies with CNA $\alpha$ and CNA $\beta 2$, but not with $\mathrm{CNA} \beta 1$. By contrast, the $\mathrm{CNB}$ regulatory subunit, was recovered to the same extent with all three $\mathrm{CN}$ isoforms (Supplementary Fig. 3a). Next, to identify CNA $\beta 1$-specific interactors which might include substrates, we turned to affinity purification coupled to mass spectrometry (AP-MS). HEK293 Flp-In T-REx cell lines expressing either FLAG-tagged-GFP, -CNA 3 trunc lacking the C-terminal tail (aa 1- 423; truncated after calmodulin binding site), -CNA $\beta 2$, or -CNA $\beta 1$ were developed (Fig. 3a and Supplementary Fig. 3b). This system has been successfully used to achieve moderate expression levels for signaling proteins and identify biologically relevant interactors for other protein phosphatases ${ }^{42-44}$, although we were unable to directly compare expression levels of the transgenes with endogenous proteins due to the low sensitivity of $\mathrm{CNA} \beta$-specific antibodies. Following immunoprecipitation of each $\mathrm{CN}$, labelfree, quantitative mass spectrometry was used to identify interactors, while comparing with FLAG-tagged-GFP control to eliminate non-specific binders. In total, 51 high confidence $\mathrm{CN}$ interacting proteins (defined as those with a bayesian false discovery rate $(\mathrm{BFDR}) \leq 1 \%$ ) were identified (Supplementary Fig. $3 \mathrm{c}$ and Supplementary Data 1). As expected, some established CNA interactors, including the $\mathrm{CNB}$ subunit (PPP3R1) and the inhibitor RCAN3, were identified with all CNA $\beta$ constructs (Fig. $3 b$ and Supplementary Fig. 3c). Of these 51 proteins, 12 were previously identified $\mathrm{CN}$-interactors and several, including BRUCE, FAM126A, and GSK3 $\beta$ contain predicted CN-binding motifs (LxVP or PxIxIT) confirming the validity of our data set ${ }^{6,7,45}$.

Excitingly, several proteins preferentially associated with $\mathrm{CNA} \beta 1$ relative to $\mathrm{CNA} \beta 2$ or $\mathrm{CNA} \beta$ trunc, i.e. spectral counts $\geq 1.5 \mathrm{x}$ more for CNA $\beta 1$ than other baits (Fig. $3 \mathrm{~b}$ ), and, consistent with CNA $\beta 1$ localization, were mostly membrane-associated, i.e.
Baculoviral IAP repeat-containing protein 6 (BIRC6/BRUCE), which localizes to the Golgi and endosomes ${ }^{46}$, PM-associated Phosphorylase B kinase regulatory subunit (PHKB), cell junction protein Liprin-Beta 1 (PPFIBP1) ${ }^{47}$, and endosomal SH3 and BAR domain-containing protein endophilin B2 (SH3GLB2) ${ }^{48}$. Strikingly, all subunits of the large PM-associated PI4KA complex were identified: EFR3B, FAM126A (Hyccin), TTC7B, and PI4KA (PI4KIIIa) (Fig. 3b, c) ${ }^{23,24,26}$. Together, these findings suggest that CNA $\beta 1$ interacts with a unique set of membrane-associated proteins which may represent CNA $\beta 1$-regulated substrates and pathways.

CNA $\beta 1$ interacts with the PI4KA complex at the plasma membrane. We further examined the CNA $\beta 1$ interaction with the PI4KA complex, which is endogenously expressed at very low levels. Therefore, we engineered a single plasmid that encodes tagged EFR3B, TTC7B, and FAM126A separated by the viral 2A linkers, T2A and $\mathrm{P} 2 \mathrm{~A}$, respectively, which are cleaved during translation to ensure balanced expression of each protein with constant stoichiometry (Supplementary Fig. 3d) ${ }^{49}$. Proper expression of each component was verified (Supplementary Fig. 3e) and expected PM localizations for EFR3B, TTC7B, FAM126A (Supplementary Fig. 3f) and co-expressed PI4KA (Supplementary Fig. 5c) were confirmed by indirect immunofluorescence. Using this expression system, we first validated preferential interaction of the PI4KA complex with CNA $\beta 1$ compared to CNA $\beta 2$ by immunoprecipitation. HEK293 Flp-In TREx cells inducibly expressing GFP-FLAG control, GFP-CNA $\beta 2$, or GFP-CNA $\beta 1$ were transfected with the EFR3B-HA, TTC7BMYC, FLAG-FAM126A-containing plasmid together with GFPPI4KA. EFR3B-HA was immunoprecipitated from cell lysates and co-purifying proteins were analyzed. As expected, GFP-PI4KA efficiently co-purified with EFR3B indicating functional complex formation (Fig. 3d, e). Supporting the AP-MS results, significantly more GFP-CNA $\beta 1$ associated with EFR3B compared to GFP$\mathrm{CNA} \beta 2$ or palmitoylation-defective GFP-CNA $\beta 1^{\mathrm{C} 2 \mathrm{~S}}$. Thus, CNA $\beta 1$ preferentially interacts with the PI4KA complex due to its unique PM localization, which is mediated by palmitoylation.

FAM126A has a putative PxIxIT motif that mediates binding to $\mathrm{CN}$. A highly conserved sequence in the intrinsically disordered C-terminal tail of FAM126A, ${ }^{512}$ PSISIT $^{517}$, which matches the consensus of the CN-binding PxIxIT motif was identified $^{6,7}$ (Fig. 4a and Supplementary Fig. 4a). To examine CN binding, we fused a 16-mer peptide containing this FAM126A sequence to GST and tested its co-purification with the recombinant, HIS-tagged $\mathrm{CN}$ heterodimer in vitro, using the PxIxIT sequence from NFATC1 as a positive control. As expected, the 
a

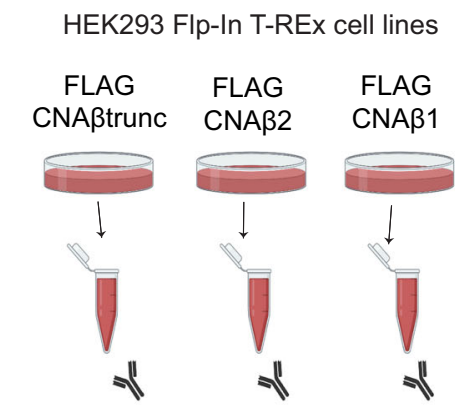

Pulldown with anti-FLAG beads

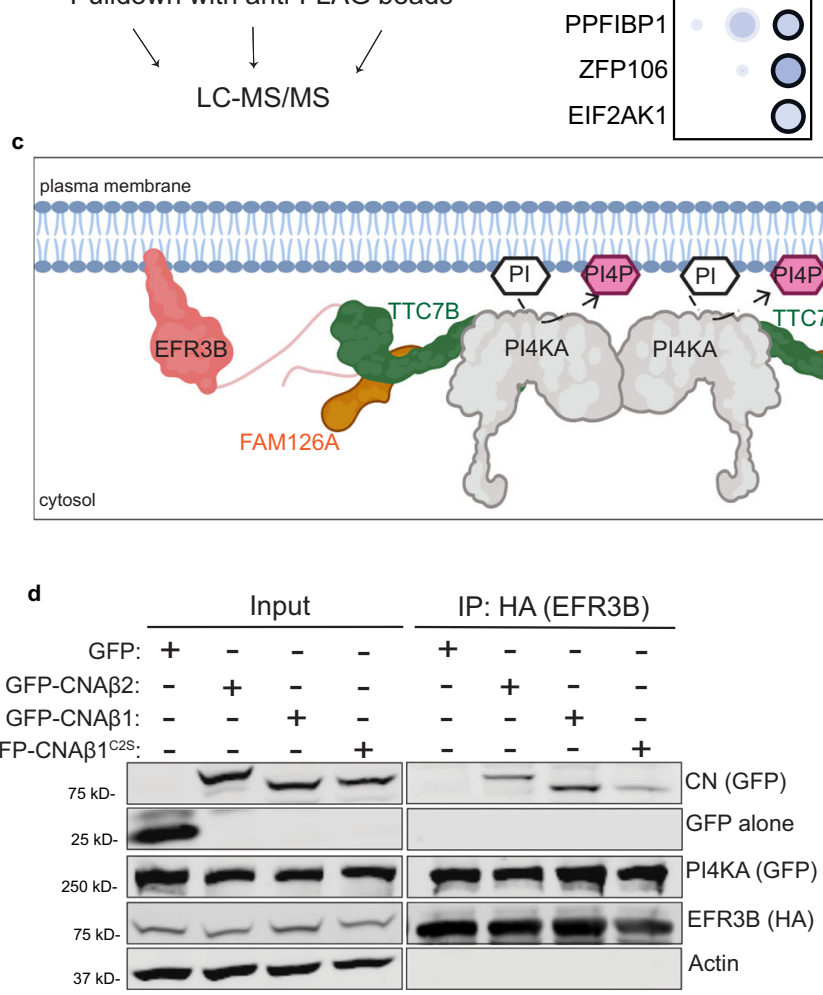

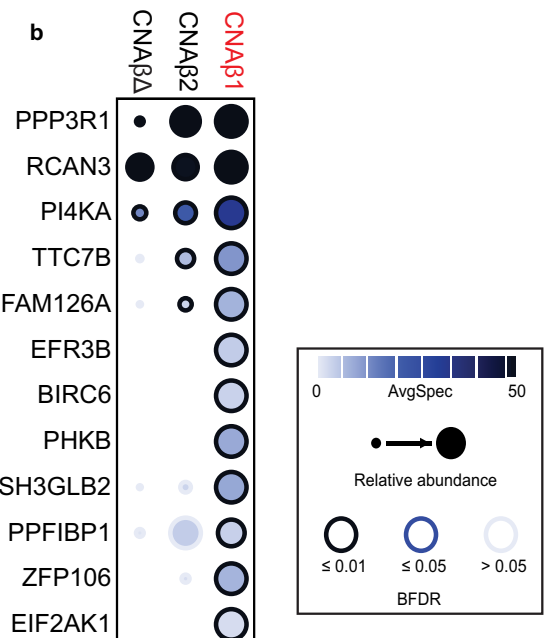


a
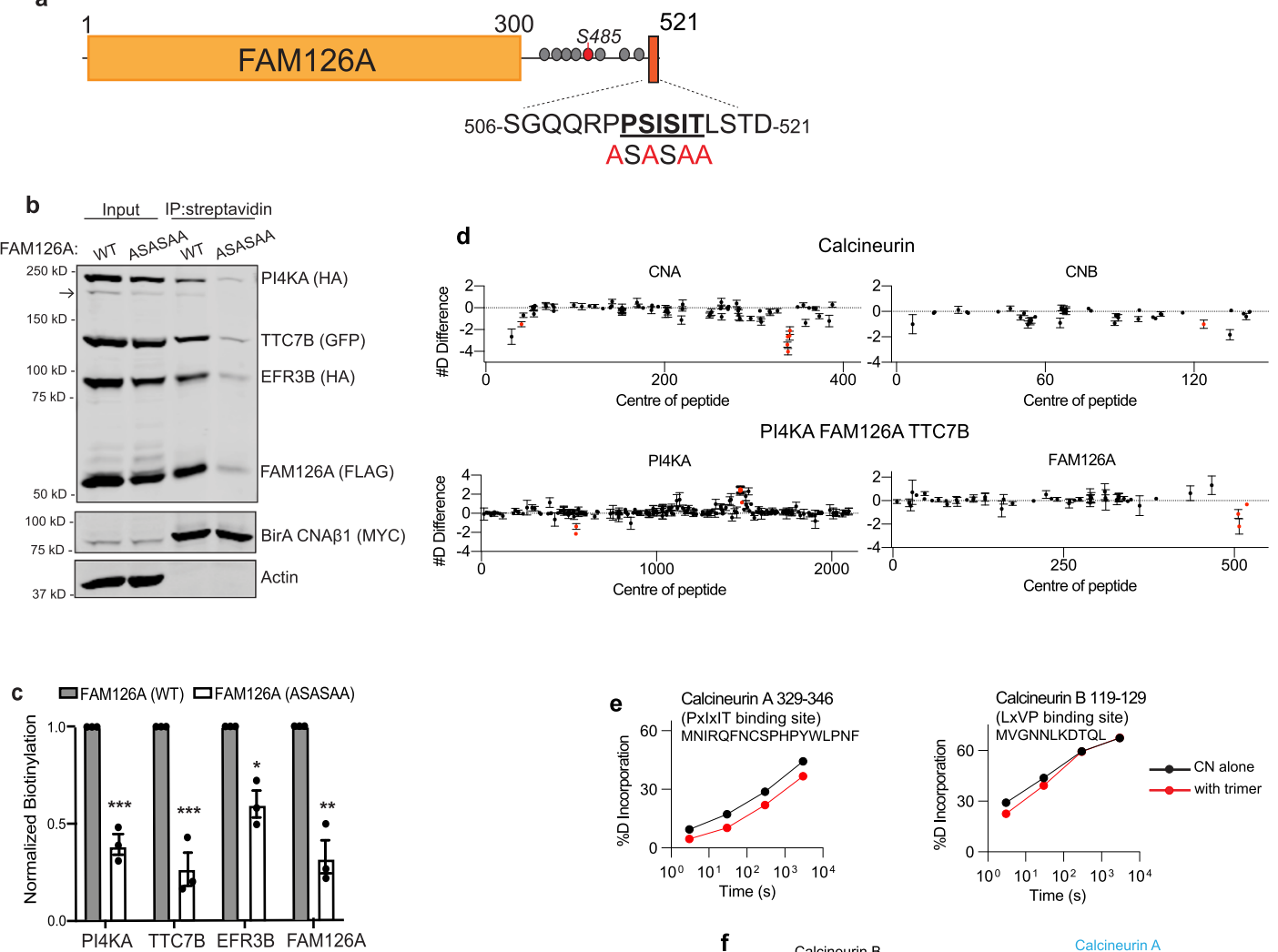

g
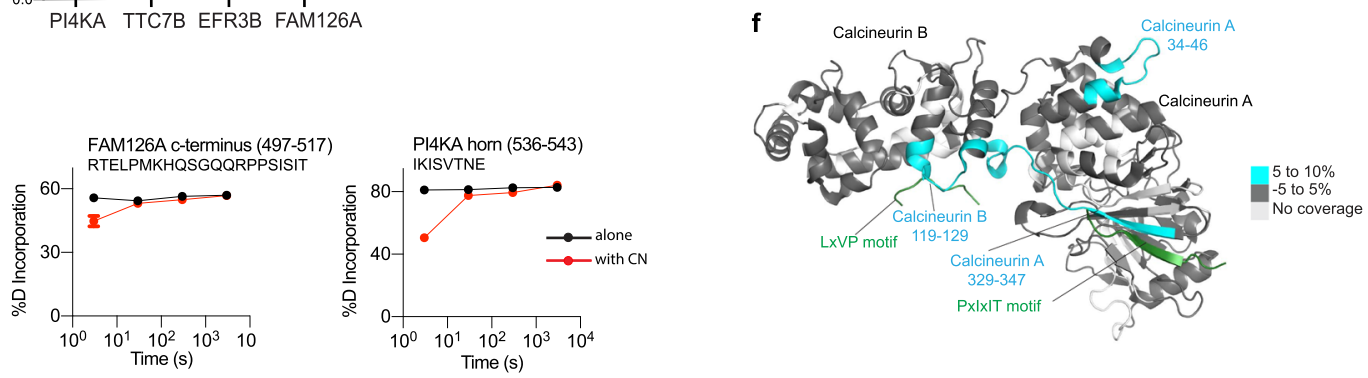

h
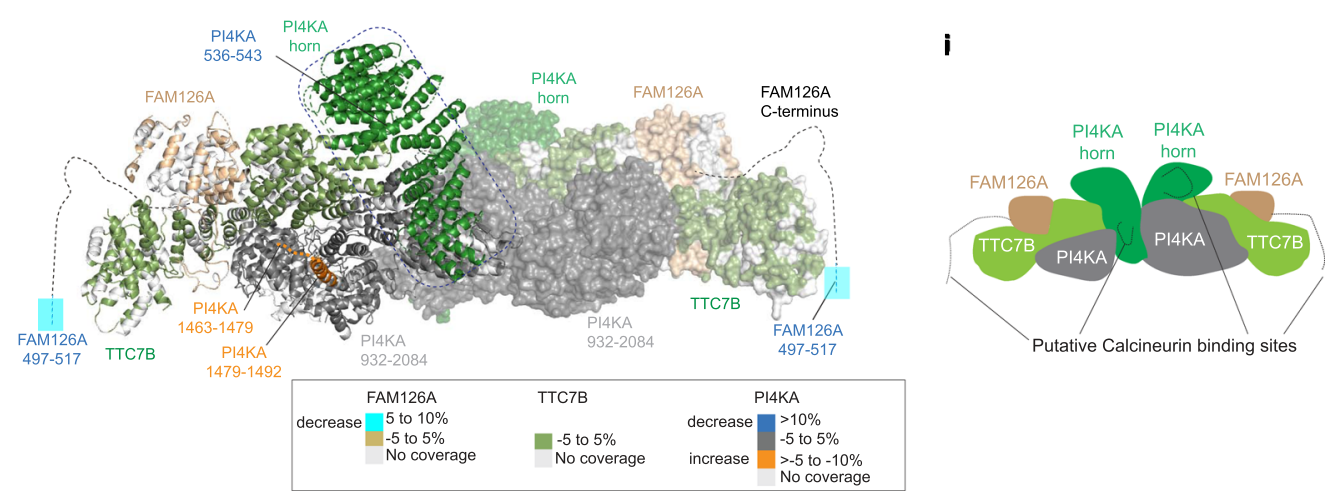

Hydrogen/deuterium exchange maps CN- PI4KA complex interaction sites. The cryo-EM structure of PI4KA-TTC7BFAM126A fails to resolve the PxIxIT-containing, unstructured, disordered C-terminal tail of FAM126A ${ }^{28}$. Therefore, to map the CN-PI4KA complex interaction and identify any conformational changes that occur upon binding, we turned to HDX-MS 29,53 . HDX-MS measures the exchange rate of amide hydrogens with deuterium-containing buffer, which sensitively probes secondary structure dynamics and solvent accessibility ${ }^{54}$. The CNA/CNB heterodimer was produced in Escherichia coli and recombinant PI4KA in complex with TTC7B and FAM126A was purified from insect cells as previously described ${ }^{29}$. The PI4KA/TTC7B/ FAM126A trimer and CNA/CNB were exposed to pulses of deuterium when incubated alone or together, with $\mathrm{CN}$ in excess over the PI4KA trimer. Localization of differences in HDX requires proteolysis into peptides, with sequence coverage for PI4KA, FAM126A, TTC7B, CNA, and CNB of $77.6 \%, 80.9 \%$, $84.2 \%, 89 \%$, and $89.3 \%$, respectively (Supplementary Table 1). Following addition of deuterium-containing buffer $\left(\mathrm{D}_{2} \mathrm{O}\right)$, reactions were quenched at indicated times $(3 \mathrm{~s}, 30 \mathrm{~s}, 300 \mathrm{~s}, 3000 \mathrm{~s})$ and the resulting shifts in mass upon deuterium incorporation were analyzed via mass spectrometry. Peptides that showed 
Fig. 4 CN-PI4KA complex interactions include a PxIxIT motif in FAM126A. a FAM126A schematic showing PxIxIT motif (PSISIT, bold)-containing peptide and mutations (ASASAA, red); phosphorylated residues (gray circles) including Ser 485 (red circle). b Representative immunoblot showing proximity-dependent biotinylation analysis of the expressed PI4KA complex containing FLAG-FAM126A (WT or ASASAA) with MYC-BirA*-CNA 1 in HeLa cells; arrow indicates uncut P2A protein ( $n=3$ independent experiments). c Biotinylation of each (as in b) quantified as respective bound signal/ MYC bound signal normalized to respective signal/Actin signal in inputs. Data show mean \pm SEM $(n=3$ independent experiments). n.s. not significant, ${ }^{\star} p=0.0037,{ }^{\star \star} p=0.0014,{ }^{\star \star \star} p=0.000262$ for PI4KA, ${ }^{\star \star \star} p=0.000886$ for TTC7B using multiple unpaired t-tests using Sidak Method. $\mathbf{d}, \mathbf{e}$ HDX data for CN (CNA/CNB) and the PI4KA/TTC7B/FAM126A trimer. ( $n=3$ independent replicates) $\mathbf{d}$ Sum of the differences in the number of deuterons incorporated for all analyzed peptides over the HDX time course shown for proteins that differ significantly in apo vs. complex state. Peptides with significant $\operatorname{HDX}(>5 \%,>0.5 \mathrm{Da}$, and an unpaired, two-tailed $t$-test $p<0.01)$ (red); central residue of each peptide is plotted. e Deuterium incorporation differences between selected CNA and CNB peptides in the presence (red) or absence (black) of PI4KA/TTC7B/FAM126A trimer are shown. f Peptides with maximum significant HDX differences in CNA/CNB upon incubation with PI4KA trimer mapped onto the structure of $\mathrm{CN}^{57}$ (PDB: 6 NUC), coloring explained in legend; PxIxIT and LxVP motifs of the NHE1 peptide (green). $\mathbf{g}$ Deuterium incorporation in FAM126A and PI4KA peptides displaying significantly decreased amide exchange in the presence (red) vs absence (black) of CN. All error bars in panels $\mathbf{d}-\mathbf{g}$ show the S.D. ( $n=3$ independent replicates), with many being smaller than the size of the point. $\mathbf{h}$ Maximum significant differences in HDX observed at any timepoint for PI4KA/FAM126A/ TTC7B trimer in the presence of CN mapped onto the structure of the PI4KA trimer28 (PDB: 6BQ1). Dotted lines: unresolved regions in the PI4KA/TTC7/ FAM126A structure; colors show differences in exchange as indicated in the legend. i Schematic of PI4KA complex showing putative CN-interacting sites. For complete dataset see Supplementary Fig. 4d, e, Supplementary Table 1 and Source Data file.

differences in amide exchange $>0.5 \mathrm{Da}$ and $>5 \%$ at any time point and had unpaired $t$-test values of $p<0.01$, across three replicates, were considered significant.

Co-incubation of CNA/CNB with the PI4KA/TTC7B/ FAM126A trimer resulted in a large decrease in HDX in the well-characterized PxIxIT-docking groove in $\mathrm{CNA}^{50}$ (aa 329-346) (Fig. 4d, e, f), consistent with the demonstrated PxIxIT-mediated FAM126A-CN interaction. The N-terminus of CNA (aa 34-36) also displayed decreased amide exchange, suggesting that previously unidentified conformational changes occur upon substrate binding (Fig. 4d, f and Supplementary Fig. 4d, e). Interestingly, a region in CNB that forms part of the LxVP-binding groove ${ }^{15}$ (aa 119-129) also showed significantly decreased amide incorporation suggesting additional, as yet unidentified, LxVP-mediated interactions between the PI4KA trimer and CN (Fig. 4d, e, f and Supplementary Fig. 4d, e). As for the PI4KA complex, while no significant changes in amide exchange were seen in TTC7B (Supplementary Fig. 4e), regions in both FAM126A and PI4KA showed significant changes in deuterium exchange in the presence of CNA/CNB. In FAM126A, exchange decreased significantly in the region containing the PSISIT sequence (aa 497-517) consistent with CN-binding to this site (Fig. 4d, g, h, i). In PI4KA an unstructured region within the a-solenoid domain (aka the horn) (aa 536-543) showed a decrease in deuterium incorporation (Fig. $4 \mathrm{~d}, \mathrm{~g}, \mathrm{~h}, \mathrm{i}$ ), indicating formation of secondary structure either from direct interaction with $\mathrm{CN}$ or as an indirect consequence of $\mathrm{CN}$ binding to FAM126A. Interestingly, this region contains a PxIxIT-like peptide sequence, "IKISVT", which may be a non-canonical CN-binding motif. In addition, a set of peptides identified in PI4KA between residues 1463-1492 showed increased amide exchange (Fig. $4 \mathrm{~d}, \mathrm{~h}$ and Supplementary Fig. $4 \mathrm{~d}, \mathrm{e}$ ) revealing a $\mathrm{CN}$-induced conformational change. Overall, these studies indicate multiple sites of contact between $\mathrm{CN}$ and PI4KA/ TTC7B/FAM126A suggestive of a regulatory interaction.

FAM126A is a CN substrate. To examine whether $\mathrm{CN}$ regulates phosphorylation of the PI4KA complex we focused on FAM126A because of its small size $(\sim 58 \mathrm{kDa})$, confirmed CN-binding motif and several known phosphorylation sites ${ }^{55}$. First, we expressed FLAG-FAM126A ${ }^{\text {WT }}$ or CN-binding defective FAM126A ASASAA, alone or together with TTC7B-MYC and EFR3B-HA and examined their electrophoretic mobility via SDS-PAGE and immunoblot analysis. Slower migrating forms of FAM126A were observed that were enhanced in FAM126AASASAA compared to FAM126A ${ }^{\text {WT }}$ (labelled PI and PII in Fig. 5a, lane 2 vs 5). Notably, these shifts were present only when FAM126A was co-expressed with other components (Fig. 5a, lane 1 vs 2 or lane 4 vs 5), especially EFR3B, the membrane anchor for the complex ${ }^{24}$ (Supplementary Fig. 5a). These slower migrating forms, indicative of hyperphosphorylation, suggest that FAM126A is phosphorylated only when associated with the PM-localized PI4KA complex i.e. by a PM-associated protein kinase, and that $\mathrm{CN}$ dephosphorylates FAM126A in a PxIxIT-dependent manner. To further analyze FAM126A phospho-regulation, we mutated several serine and threonine residues observed to be phosphorylated ${ }^{55}$ to the non-phosphorylatable amino acid alanine. Remarkably, mutating serine 485 (FAM126A ${ }^{\text {S485A }}$ ) altered mobility shifts in FAM126A, eliminating PII and reducing PI (Fig. 5a, lane 2 vs 3 and lane 5 vs 6 ), suggesting that Ser485 is one target of phosphorylation and that additional sites likely contribute to the observed shifts. To analyze the phosphorylation status of Ser485 in FAM126A, we generated a phospho-specific antibody, anti-pFAM126A S485. The specificity of this antibody is demonstrated by analyses of HeLa cells expressing FAM126A mutants (S485A, ASASAA, or ASASAA+S485A) with or without EFR3B and TTC7B coexpression, where this antibody specifically recognized both slower-migrating FAM126A forms (PI and PII, Fig. 5a). Notably, no signal was detected for FAM126A ${ }^{\text {S485A }}$ or when FAM126A was expressed alone, and the total signal was significantly higher for FAM126A ASASAA compared to FAM126A ${ }^{\mathrm{WT}}$. Moreover, indirect immunofluorescence using anti-pFAM126A S485 antibody showed enriched signal at the PM, further indicating that FAM126A is phosphorylated when the PI4KA complex is PMassociated (Supplementary Fig. 5b).

Next, we used anti-pFAM126A S485 to probe FAM126A phosphorylation in cells under different signaling conditions. Although direct phosphorylation of the PI4KA complex has not been demonstrated, a recent study identified PKC as a possible regulator of this complex and showed that PMA activates PI4P production at the PM which is blocked by BIM, a PKC inhibitor $^{22}$. Therefore, we monitored FAM126A phosphorylation with anti-pFAM126A S485 under similar conditions by treating cells that co-expressed FLAG-FAM126A (WT or ASASAA mutant), TTC7B and EFR3B upon treatment with combinations of a CN inhibitor (FK506), a PKC activator (PMA) and a PKC inhibitor (BIM). By examining the total intensity of antipFAM126 S485 signal (forms PI and PII), we made the following observations (Fig. 5c): First, for cells expressing FAM126A WT, addition of FK506 significantly increased Ser485 phosphorylation under all conditions (alone or together with PMA, PMA+BIM). Second, compared to FAM126A ${ }^{\mathrm{WT}}$, cells expressing 
a

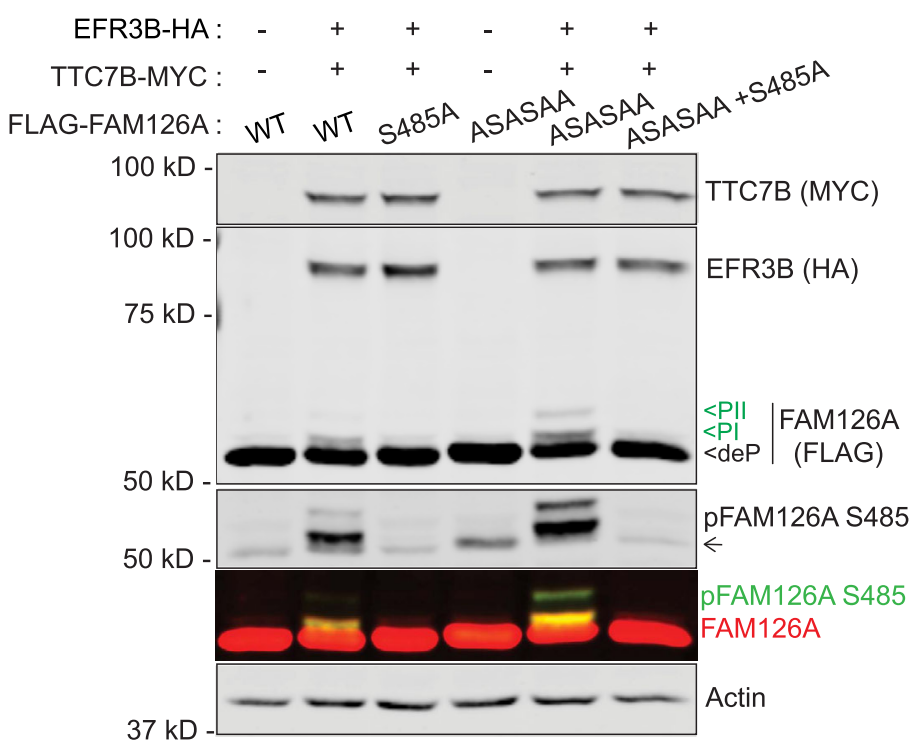

b
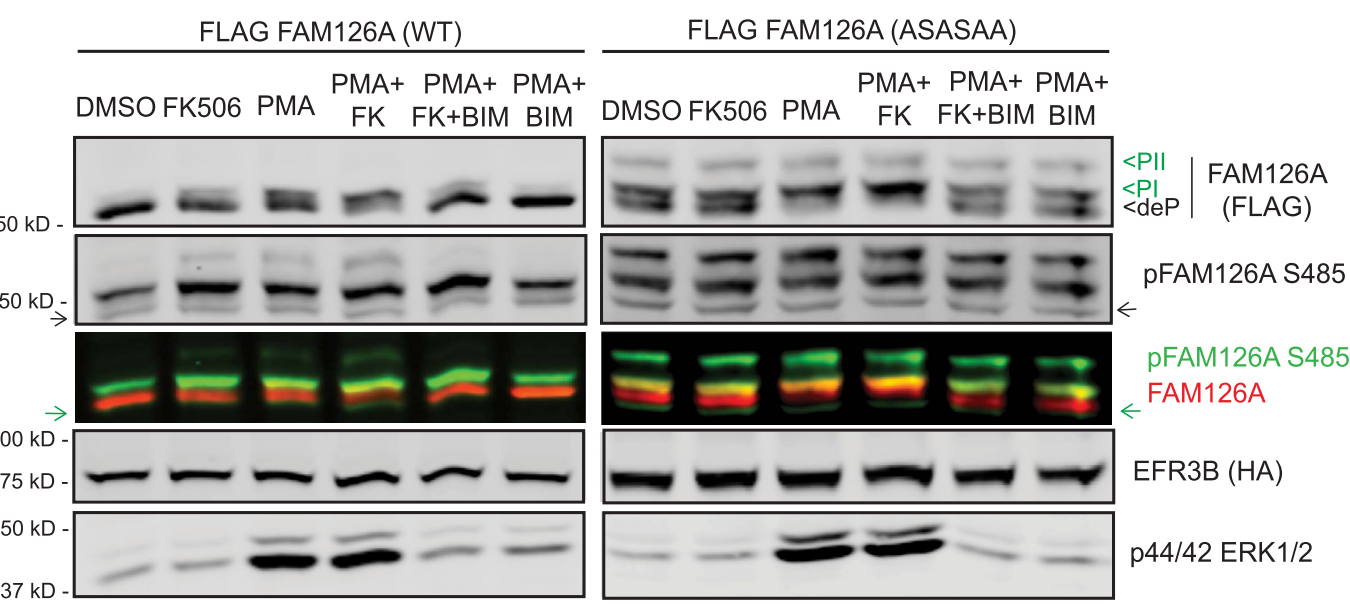

c

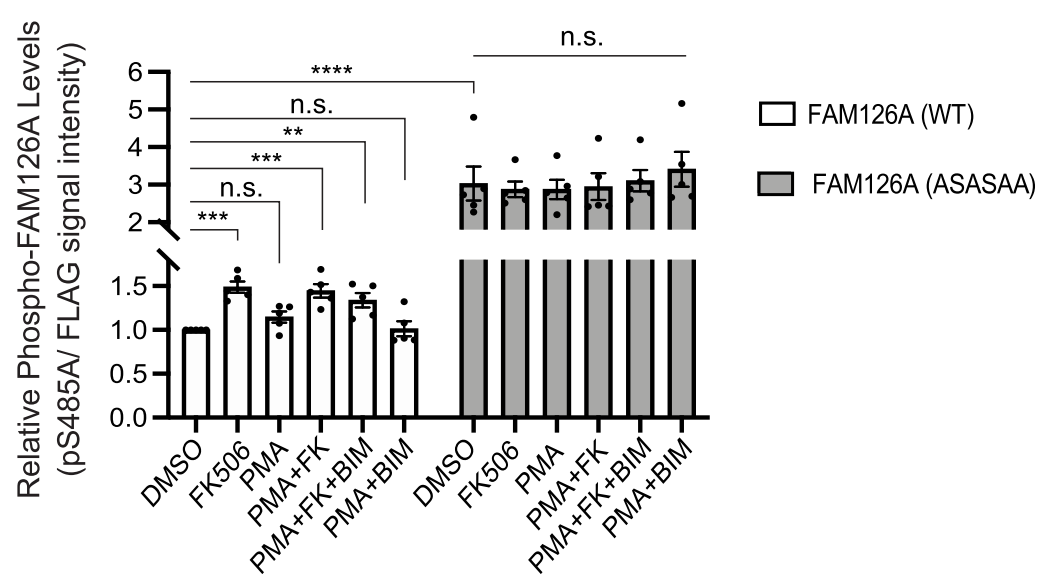

FAM126A ASASAA showed higher levels of Ser485 phosphorylation under all conditions and inhibiting CN with FK506 had no further effect as expected for this $\mathrm{CN}$-binding impaired mutant. Together, these findings show that $\mathrm{S} 485$ phosphorylation is $\mathrm{CN}$ regulated. Furthermore, addition of PMA did not enhance S485 phosphorylation which was detected under basal conditions, suggesting that this site is constitutively phosphorylated. Next, we focused on shifts in electrophoretic mobility of FAM126A (Fig. 5b). Interestingly, for both FAM126A proteins (WT and ASASAA mutant), treatment with PMA caused an electrophoretic shift from the dephosphorylated form (deP) to PI, likely due to phosphorylation of residues other than Ser485. Importantly, this PMA-induced shift was suppressed by BIM indicating PKC dependence. In sum, these findings demonstrate PxIxIT- 
Fig. 5 FAM126A is a CN substrate. a Representative immunoblot showing electrophoreticmobility shifts observed for FLAG-FAM126A when expressed in HeLa cells. Lysates of cells expressing FLAG-FAM126A (WT, S485A, ASASAA, or ASASAA+S485A) in the presence or absence of EFR3B-HA/ TTC7BMYC were analyzed using anti-MYC, anti-HA, anti-FLAG (red bands), and phospho-specific pFAM126A S485A (green bands) antibodies. PI and PII phosphorylated states, deP dephoshorylated state ( $n=3$ independent experiments). b Representative immunoblot showing analysis of FLAG FAM126A (WT or ASASAA) phosphorylation status in HeLa cells co-expressing FLAG-FAM126A, TTC7B-MYC, and EFR3B-HA across indicated treatments: DMSO (vehicle), FK506 (FK, CN-inhibitor), PMA (PKC activator), BIM (PKC inhibitor) using anti-FLAG (red), anti-HA and anti-pFAM126A S485A (green) antibodies. PKC activation was assessed by phosphorylation of the downstream substrate, ERK using anti-p44/42 ERK 1/2 antibody. Arrows denote nonspecific antibody background. ( $n=5$ independent experiments) c FAM126A phosphorylation at Ser485 (from b) was quantified as the ratio of total pFAM126A S485 signal/ total FLAG signal relative to DMSO-treated FLAG-FAM126A WT signal ratio. Data are mean \pm SEM $(n=5$ independent experiments). ${ }^{\star \star} p=0.0081,{ }^{\star \star \star} p<0.001$ ( $p=0.0002$ for DMSO vs FK506; $p=0.0005$ for DMSO vs PMA + FK), ${ }^{\star \star \star \star} p<0.0001$, n.s. (non-significant): $p=0.53$ for DMSO vs PMA; $p>0.99$ for the rest, calculated using one-way ANOVAs with Dunnett's multiple comparison tests.

dependent regulation of FAM126A phosphorylation at Ser485 by $\mathrm{CN}$ in vivo and establish FAM126A as a CN substrate. These data also reveal PMA-induced phosphorylation of FAM126A, at a distinct site, likely by PM-localized PKC, which might be the molecular basis of the reported regulatory role for PKC in PM PI4P synthesis ${ }^{22}$.

CN regulates PI4P synthesis by the PI4KA complex. Having shown that $\mathrm{CN}$ interacts with the PI4KA complex and that FAM126A is a CN substrate, we next investigated whether $\mathrm{CN}$ regulates the assembly and/or activity of this complex. First, we examined interaction of the cytosolic heterotrimer, PI4KA/ TTCB/FAM126A, with the membrane anchor EFR3B in the presence of FAM126A $\mathrm{WT}^{\mathrm{WT}}$ or CN-binding defective FAM126A $\mathrm{A}^{\mathrm{A}-}$ SASAA. Immunopurification of EFR3B-HA showed the same levels of co-purifying GFP-PI4KA, TTC7B-MYC or FLAGFAM126A with FAM126A ${ }^{\mathrm{WT}}$ or FAM126A ASASAA (Fig. 6a, b). Furthermore, indirect immunofluorescence analyses of these cells verified that each component, especially GFP-PI4KA, localized to the PM with either FAM126A ${ }^{\mathrm{WT}}$ or FAM126A ASASAA, indicating that the complex formed properly (Supplementary Fig. 5c). Thus, no $\mathrm{CN}$-dependent regulation of complex formation via FAM126A was observed.

Next, we explored whether CN regulates PM PI4P synthesis carried out by the PI4KA complex using a previously established bioluminescence resonance energy transfer (BRET) assay that monitors PI4P levels at the PM in live cells during signaling 22 . For this assay, the energy donor (luciferase) is fused to the PI4P binding domain, P4M, of the Legionella SidM protein ${ }^{56}$, the energy acceptor (Venus) is attached to the PM-targeting sequence from Lck (first ten amino acids, L10) (Fig. 6c), and both proteins are co-expressed with the Gq-coupled muscarinic receptor, $M_{3} R$, in HEK293T cells. As previously reported, PM PI4P levels transiently increased in control cells (blue lines, Fig. 6d) following addition of the $\mathrm{M}_{3} \mathrm{R}$ ligand, carbachol $\left(10^{-7} \mathrm{M}\right)$, due to activation of the PI4KA complex ${ }^{22}$. Excitingly, pre-treatment of these cells with CN inhibitors, FK506 $(1 \mu \mathrm{M})$ or CsA $(10 \mu \mathrm{M})$, significantly reduced the level of PI4P produced (red lines, Fig. $6 \mathrm{~d}$ ) consistent with our hypothesis that $\mathrm{CN}$ regulates PI4KA complex activity under $\mathrm{Ca}^{2+}$ signaling conditions.

In summary, our findings lead us to propose the following model: Signaling from a Gq-coupled GPCR generates an intracellular $\mathrm{Ca}^{2+}$ signal that activates $\mathrm{CN}$, and likely $\mathrm{PKC}$, which in turn stimulate the PI4KA complex at the PM to promote $\mathrm{PI} 4 \mathrm{P}$ replenishment and thus generating $\mathrm{PI}(4,5) \mathrm{P}_{2}$ pools required for sustained signaling (Fig. 7). Our work identifies CNA $\beta 1$ as an interaction partner of the PI4KA complex, shows that $\mathrm{CN}$ inhibitors alter PI4P production at the PM during signaling, and warrants further investigation into the phosphorylation state of complex components, especially FAM126A and PI4KA, through which $\mathrm{CN}$ might be regulating PI4KA activity.

\section{Discussion}

In this study we aimed to discover $\mathrm{CN}$ signaling pathways that are regulated by the naturally occurring but understudied $\mathrm{CN}$ isoform, CNA $\beta 1$, which is conserved among vertebrates and broadly expressed ${ }^{10-13}$. This isoform differs from canonical CNA $\beta 2$ only in its $40 \mathrm{C}$-terminal residues ${ }^{10}$, which confer distinct enzymatic regulation to $\mathrm{CNA} \beta 1$ through an LxVP-type autoinhibitory sequence $(\mathrm{LAVP})^{12}$. Here we show that the CNA $\beta 1$ tail is dually palmitoylated, making $\mathrm{CNA} \beta 1$ the only known form of $\mathrm{CN}$ that directly associates with the PM and Golgi. By contrast, canonical $\mathrm{CN}$ isoforms access only select PM proteins that either contain CN-binding sites in their cytosolic domains (e.g. NHE1, TRESK) ${ }^{57,58}$ or associate with membrane-anchored scaffolds such as AKAP7959. This unique localization determines CNA $\beta 1$ substrate specificity including its interaction with all four members of the protein complex that synthesizes the critical phospholipid, PI4P at the PM. We demonstrate that FAM126A, the regulatory component of this complex, is phosphorylated at the PM, directly binds $\mathrm{CN}$, and contains at least one $\mathrm{CN}$ regulated phosphorylation site. These findings led us to discover a hitherto unknown role for $\mathrm{CN}$ in regulating PI4P synthesis at the PM during GPCR signaling. The CNA $\beta 1$ isoform is ideally positioned to carry out this regulation.

Our finding that CNA $\beta 1$ is dynamically palmitoylated has several interesting implications for its regulation in vivo. First, the ability of CNA $\beta 1$ to access membrane-associated substrates and hence carry out its functions may be controlled by the palmitoyl transferases (DHHCs) and depalmitoylases that act on it, as has been shown for other signaling enzymes including RAS and $\operatorname{LCK}^{31,39,60,61}$. Second, we speculate that palmitoylation-driven binding of the autoinhibitory CNA $\beta 1$ tail to membranes may be necessary to fully activate this variant which is only partially activated by $\mathrm{Ca}^{2+}$ and $\mathrm{CaM}$ in vitro ${ }^{12}$. Thus, examining the enzymes that modify CNA $\beta 1$ lipidation will be key for understanding how CNA $\beta 1$ is controlled physiologically. Here we show that a membrane-localized thioesterase ABHD17A, which regulates $\mathrm{H}$ - and $\mathrm{N}-\mathrm{RAS}^{39}$, also catalyzes the depalmitoylation of CNA $\beta 1$ causing it to redistribute from the PM to the cytosol and the Golgi. Recent work indicates that the ABHD17 family of depalmitoylases specifically targets $\mathrm{PM}$-associated proteins ${ }^{38}$, although mechanisms controlling their activity are yet to be identified. Furthermore, determining which of the 23 DHHCs encoded in human genome act on CNA $\beta 1$ may provide insights into where and when palmitoylation takes place, as these enzymes exhibit distinct patterns of localization and regulation ${ }^{31}$. In sum, our findings lay the groundwork for further investigation into the role of dynamic palmitoylation in controlling CNA $\beta 1$ localization and/or enzymatic activity, which may also provide tools to specifically regulate its functions.

Our investigations identify $\mathrm{CN}$ as a regulator of the PI4KA complex composed of PI4KA, TTC7B, FAM126A and EFR3B, and highlight major gaps in our knowledge of how this important 
a

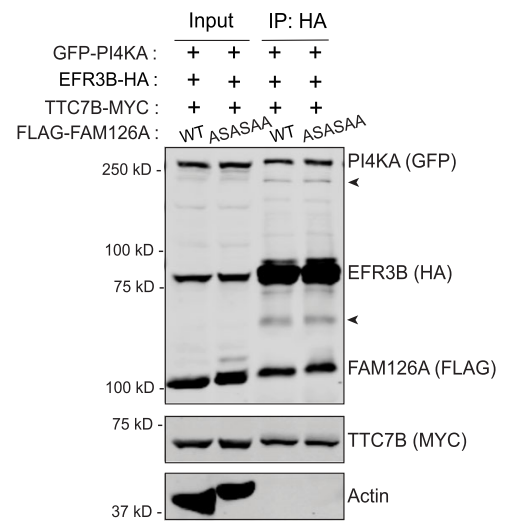

b
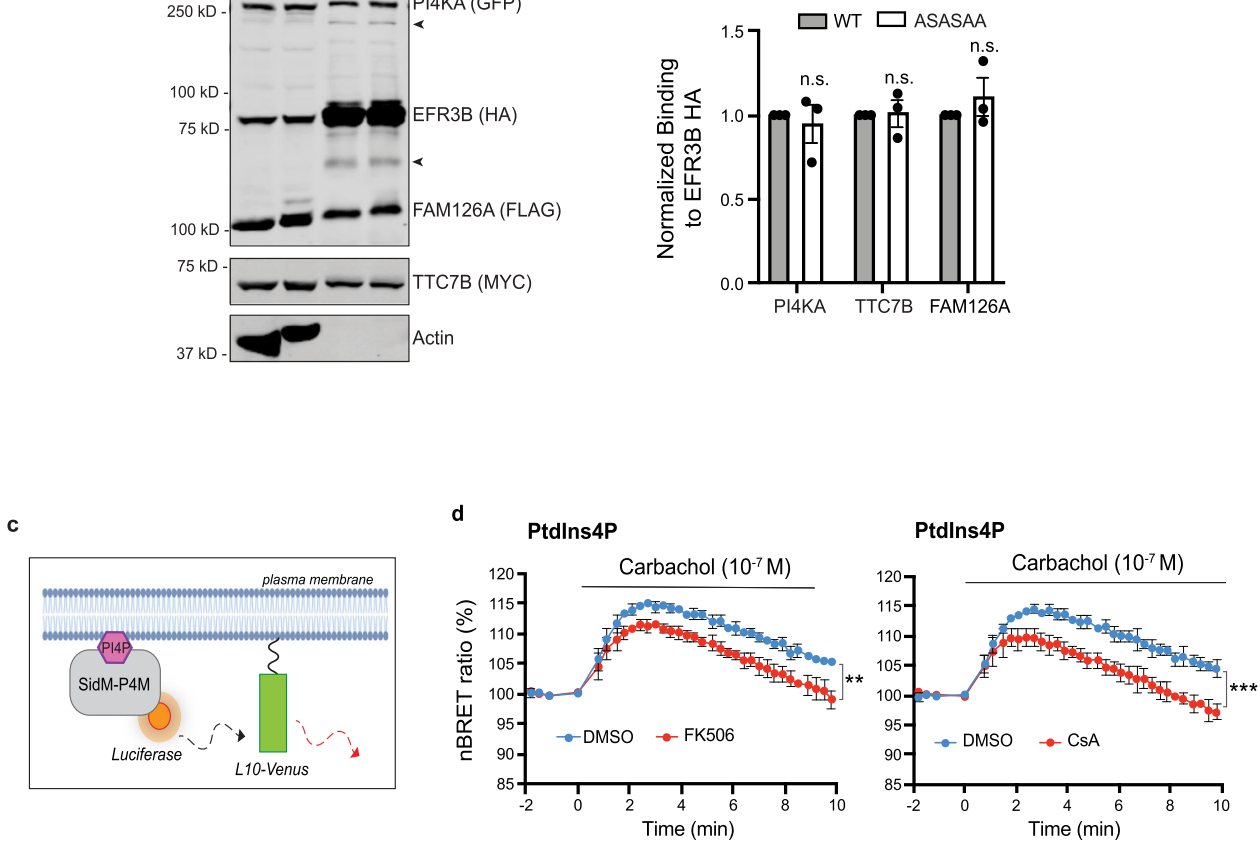

Fig. 6 CN regulates PI4P synthesis by the PI4KA complex. a Representative immunoblot showing analysis of the PI4KA complex components in anti-HA immunoprecipitates of HeLa cells expressing GFP-PI4KA, EFR3B-HA/TTC7B-MYC with WT or ASASAA mutant FLAG-FAM126A ( $n=3$ independent experiments). Arrow points to the uncut P2A form. Arrowhead denotes non-specific antibody bands. $\mathbf{b}$ Co-purification of each component with EFR3B-HA (from a) is quantified as respective signals/EFR3B-HA signal in bound fractions normalized to respective signals/Actin signal in input fractions. Data are the mean $\pm \operatorname{SEM}(n=3$ independent experiments). n.s. not significant using multiple, unpaired, two-tailed $t$-tests. $\mathbf{c}$ Cartoon representation of the BRET pair used in experiments shown in d. PI4P binding domain of the Legionella SidM protein (SidM-P4M, gray) attached to Renilla Luciferase (orange) as the donor and Venus (green), targeted to the PM using the first 10 amino acids of Lck, L10, as the acceptor. d Normalized BRET ratios reflecting changes in PM PI4P levels in response of carbachol stimulation $\left(10^{-7} \mathrm{M}\right.$ ) in HEK293T cells transiently expressing muscarinic receptor, $\mathrm{M}_{3} \mathrm{R}$, pre-treated with DMSO (blue), or CN inhibitors (red): FK506 (1 $\mu \mathrm{M})$ (left) and Cyclosporin A, CsA (10 $\mu \mathrm{M})$ (right) for $1 \mathrm{hr}$. Data shown are mean \pm SD ( $n=4$ independent experiments). ${ }^{* *} p=0.0063,{ }^{* * *} p=0.0004$ using Kolmogorov-Smirnov test.

complex is regulated. Production and maintenance of PM PI4P levels are physiologically critical as evidenced by the wide range of diseases caused by mutations in complex components ranging from neurological (PI4KA), immune and gastrointestinal (TTC7) defects, to hypomyelination and congenital cataracts (FAM126A) 24,28 . Phosphorylation regulates assembly of the PI4KA complex in yeast; however, in mammals, little is known about how the assembly or activity of this complex is modulated. Our interaction studies, including HDX-MS analysis, uncovered potential contacts between $\mathrm{CN}$ and multiple PI4KA complex members, and confirmed direct binding to a PxIxIT motif in the C-terminal tail of FAM126A. This tail is completely unstructured and shows no interaction with TTC7B or PI4KA ${ }^{24}$, but inhibits PI4KA activity in vitro through an unknown mechanism ${ }^{29}$. Our results provide insights into this regulation by demonstrating that $\mathrm{CN}$ binds to and modulates the phosphorylation of at least one site in the FAM126A tail (Ser485) in cells, which, based on our findings, is phosphorylated by an unidentified kinase that is active at the PM under basal conditions. Computational analysis failed to predict any likely candidate kinases for this site (NetPhos $3.1)^{62}$, however up to $10 \%$ of human kinases localize to the PM, including many that are uncharacterized ${ }^{63}$. Further studies are required not only to identify relevant kinases, but also to comprehensively map CN-regulated phosphorylation sites in PI4KA complex members and assess the functional consequences of these modifications.
Lastly, our discovery that $\mathrm{CN}$ inhibitors reduce PI4P production at the PM induced during $\mathrm{Ca}^{2+}$ signaling from Gq-coupled GPCRs suggests that a positive feedback loop exists through which PKC and $\mathrm{CN}$ (presumably $\mathrm{CNA} \beta 1$ ), regulate the phosphorylation of the PI4KA complex to stimulate its activity and ensure a continued supply of PI4P, the precursor of $\mathrm{PI}(4,5) \mathrm{P}_{2}$ (Fig. 7). Evidence for PKC involvement in this stimulation ${ }^{22}$ is consistent with the $\mathrm{CN}$-independent, PKC-regulated phosphorylation-shift we observed in FAM126A (Fig. 5b). Rigorously testing this model, however, is challenging due to the complete lack of knowledge about how this large, minimally expressed complex that apparently undergoes extensive allosteric rearrangements ${ }^{29}$, is regulated in cells. To date, our attempts to identify changes in PI4P levels or synthesis rates caused by mutations in the FAM126A PxIxIT site or Ser485 have been unsuccessful using overexpression of the proteins. This could be due to limitations in the experimental set up (HEK293T cells overexpressing all PI4KA complex members), or because solely altering FAM126A may not be sufficient to perturb $\mathrm{CN}$ dependent regulation of the complex. Regardless, our work establishes that CNA $\beta 1$ preferentially interacts with the PI4KA complex at the PM and suggests that FAM126A is a direct substrate of CNA $\beta 1$.

Insights into the physiological functions of $\mathrm{CNA} \beta 1$ come from studies that overexpress or more recently, delete the CNA $\beta 1$ isoform in mice $11,16,18,19$. These knock-out mice are viable, but 


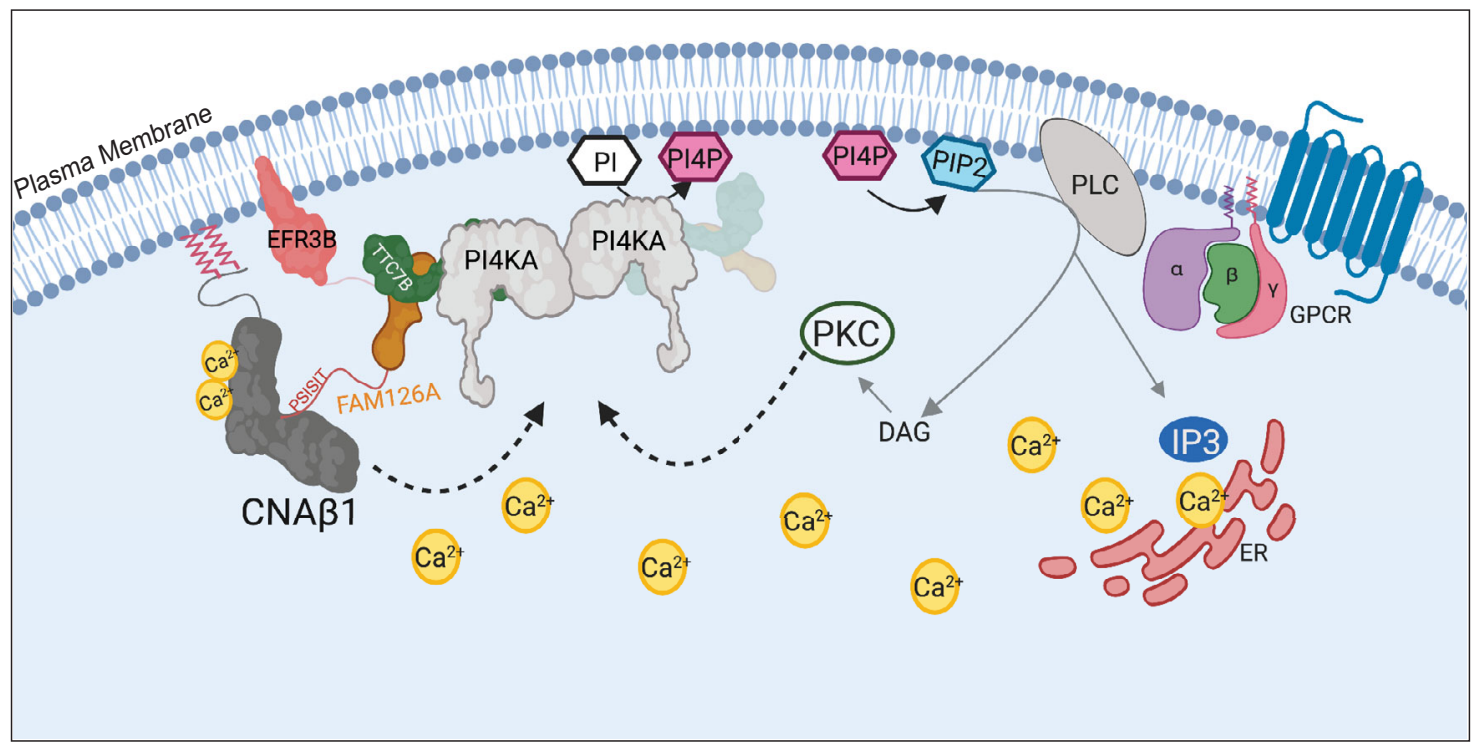

Fig. 7 Model for CNAß1 mediated regulation of the PI4KA complex that promotes PI4P synthesis at the PM during GPCR signaling. Ligand binding and subsequent PLC-mediated cleavage of $\mathrm{PI}(4,5) \mathrm{P}_{2}$ generates DAG and IP3, which causes $\mathrm{Ca}^{2+}$ release from the ER. This increase in intracellular Ca2+ activates $C N$ (CNA 1 1), and PKC, which in turn regulate the PI4KA complex at the PM to promote PI4P and PI(4,5) $P_{2}$ replenishment. See text for details. Schematic generated using BioRender.com.

develop cardiac hypertrophy, possibly due to disruptions in mTORC2/AKT signaling and serine one-carbon metabolism ${ }^{18}$. However, the precise molecular mechanisms underlying these pathologies and whether any of these phenotypes relate to PI4KA complex regulation remain to be determined. Notably, some reports indicate that $\mathrm{mTORC} 2$ activity toward AKT takes place at the PM and depends on the $\mathrm{PH}$-domain containing targeting subunit, mSIN1, which binds to phosphoinositides ${ }^{64-66}$. Furthermore, the interactors identified here suggest that CNA $\beta 1$ regulates multiple substrates throughout the body. Comprehensive identification of these targets as well as the regulatory mechanisms that control CNA $\beta 1$ activity in vivo promise to shed light on $\mathrm{Ca}^{2+}$ and $\mathrm{CN}$-regulated pathways and their possible perturbation in patients undergoing long term treatment with $\mathrm{CN}$ inhibitors, CsA or FK506/Tacrolimus.

\footnotetext{
Methods

Sequence alignments. ClustalW was used to create all sequence alignments using Jalview $^{67}$. The following species are used in Fig. 1b: Homo sapiens (human, Q5F2F8), Pan troglodytes (chimpanzee, A0A2J8NUG2), Sus scrofa (pig, A0A480QFW6), Desmodus rotundus (Bat, K9ISS2), Mus musculus (mouse, Q3UXV4), Callorhinchus milii (ghost shark, V9KGC1), and Xenopus tropicalis (western clawed frog, A0A6I8R6A9). The following species are used for Supplementary Fig. 4a: Homo sapiens (human, Q9BYI3), Gorilla gorilla (gorilla, A0A2I2YR80), Macaca mulatta (monkey, H9ZEG3), Sus scrofa (pig, I3LJX1), Felis catus (cat, M3WEC3), Bos taurus (bovine, E1BFZ6), Mus musculus (mouse, Q6P9N1), Gallus gallus (chicken, Q5ZM13), Xenopus tropicalis (western clawed frog, F7EHL4), Callorhinchus milii (ghost shark, A0A4W3JDV9), and Danio rerio (zebrafish, Q6P121).
}

Cell culture and transfection. HeLa, COS-7, and HEK 293T cells were grown at $37^{\circ} \mathrm{C}$ in a $5 \% \mathrm{CO}_{2}$ atmosphere in cell culture medium (Dulbecco's modified Eagle's medium, DMEM) (CA 10-013, Sigma-Aldrich) supplemented with $10 \%$ fetal bovine serum (FBS, Benchmark ${ }^{\mathrm{TM}}$ Gemini Bio Products). Cells were transfected as indicated in each experiment using jetOPTIMUS (VWR) as per the manufacturer's instructions. HeLa cells were gifts from the Skotheim lab. COS-7 cells were purchased from ATCC (CRL-1651). Cells were validated via STR profiling ${ }^{68,69}$ (see Source Data file).

Stable cell line generation. Human [taxid:9606] cells [Flp-In T-REx 293 cells], obtained from Gingras Lab, were transfected in a six-well format with $0.2 \mu \mathrm{g}$ of tagged DNA in pcDNA5 FRT TO vector and $2 \mu \mathrm{g}$ pOG44 (OpenFreezer V4134), using lipofectamine 2000 (Life Technologies), according to the manufacturer's instructions. On day 2, cells were trypsinized, and seeded into $10 \mathrm{~cm}$ plates. On day 3 , the medium is replaced with DMEM containing $5 \%$ fetal bovine serum, $5 \%$ calf serum, 100 units $/ \mathrm{ml}$ penicillin/streptomycin, $3 \mu \mathrm{g} / \mathrm{ml}$ blastocidin (RPI), and $200 \mu \mathrm{g} /$ $\mathrm{ml}$ hygromycin (Sigma-Aldrich). Medium was replaced every 2-4 days until nontransfected cells died and isolated clones were $\sim 1-2 \mathrm{~mm}$ in diameter (13-15 days). Pools of cells were generated by trypsinization of the entire plate and replating in fresh selection medium. Pools were amplified to one $15 \mathrm{~cm}$ plate. From this plate, cells were trypsinized (volume $=8 \mathrm{ml}$ ) and replated in five $15 \mathrm{~cm}$ plates. A frozen stock was generated from the plate when cells reached $\sim 80 \%$ confluence.

Plasmids. DNAs encoding the human CNA $\beta 1$ (1-496), CNA $\beta 2$ (1-524) were subcloned into a pcDNA5 expression vector which encodes an N-terminal FLAG tag or GFP tag. FAM126A cDNA received from Addgene, was subcloned into pcDNA5 with N-terminal FLAG tag, between BamHI and XhoI sites. Variants of CNA $\beta 1$ $\left(\mathrm{CNA} \beta 1^{\mathrm{C} 483 \mathrm{~S}}\right.$, CNA $\beta 1^{\mathrm{C} 493 \mathrm{~S}} \mathrm{CNA} \beta 1^{\mathrm{C} 2 \mathrm{~S}}$ ) and FAM126A (FAM126A ${ }^{\mathrm{ASASAA}}$,

FAM126A ${ }^{\text {S485A }}$, FAM126A ASASAA+S485A) were generated using the Quickchange (Agilent) site-directed mutagenesis kit. Plasmids containing the DNA encoding ABHD17A (WT and S190A mutant), APT1, APT2, and Venus-RIT were gifts from the Conibear lab. CNA/CNB plasmid (residues 2-391 of human CNA alpha isoform and human CNB isoform 1) tandemly fused in pGEX6P3 (which encodes $\mathrm{N}$ -terminal GST tag) for protein purification and use in HDX-MS experiments were cloned as described before ${ }^{6}$. 6x His-CNA (residues 1-391 of human CNA alpha isoform and human CNB isoform 1 tandemly fused in, p11 vector) used in in vitro peptide binding assays, was cloned as described before ${ }^{15}$. His-CN NIR $\left({ }^{330} \mathrm{NIR}^{332}\right.$ -AAA mutations) generated using site-directed mutagenesis using His-CN WT as template. Plasmids encoding human PI4KIIIa were gifts of the Balla lab. Plasmids encoding human EFR3B and TTC7B, both C-terminally tagged, were gifts of the De Camilli lab. EFR3BHA_T2A_TTCBMYC (or GFP)_P2A_FLAG FAM126A plasmid was generated in between HindIII and NotI sites of pcDNA3.1 vector. The DNA sequence that encodes viral T2A (GSGEGRGSLLTCGDVEENPGP) was subcloned to the $5^{\prime}$ end of the EFR3B-HA sequence, TTC7B-MYC was then subcloned in frame to the T2A sequence. FLAG-FAM126A with DNA sequence encoding for P2A (GSGATNFSLLKQAGDVEENPGP) was cloned in frame, at the $5^{\prime}$ end of the TTC7B-MYC sequence. Primers used to generate plasmids can be found in Supplementary Table 2.

Antibodies. Commercial antibodies used, along with their working dilutions, are indicated in the methods section for each experiment. The phosphospecific antibody against Serine 485 site in FAM126A was manufactured by 21 st Century Biochemicals as follows: a peptide corresponding to the sequence Hydrazine-AhxANRFSAC[pS]LQEEKLI-amide was manufactured by Fmoc chemistry, HPLC purified to $>90 \%$, and its mass and sequence were verified by nanospray MS and CID MS/MS, respectively. The peptides, along with carrier proteins and adjuvant, were injected into New Zealand White rabbits using an initial CFA injection, followed by IFA injections. A production bleed was then taken from each of the rabbits. Sera were passed multiple times over a hydrazine reactive resin which was linked to the immunogen peptides, then rinsed with both salt and phosphate 
buffers. The antibody fractions were collected using an acidic elution buffer and immediately neutralized before a two-stage dialysis into PBS buffer, $\mathrm{pH}$ 7.2. The antibody concentration was determined using a spectrophotometer (A280). The purified antibodies were then passed multiple times over a hydrazine reactive resin, which is linked with the unmodified peptides (those not injected). These immunodepletion steps were done to remove any non-specific/phospho-independent antibodies. The final antibodies were then buffered in a PBS $/ 50 \%$ glycerol buffer, $\mathrm{pH} 7.2$ and the final concentration was calculated using a spectrophotometer (A280).

Immunofluorescence, microscopy, and image analysis. HeLa or COS-7 cells were grown on $12 \mathrm{~mm}$, \#1.5H glass coverslips (ThorLabs). In all, $24 \mathrm{~h}$ posttransfection, cells were washed with $1 \mathrm{X}$ PBS and fixed in $4 \%$ paraformaldehyde (PFA) solution (diluted from 16\% PFA, Electron Microscopy Sciences) in PBS for $15 \mathrm{~min}$. Cells were washed thrice with PBS and permeabilized for $5 \mathrm{~min}$ in block buffer (1x PBS with $0.2 \mathrm{M}$ Glycine, $2.5 \% \mathrm{FBS}$ ) with $0.1 \%$ Triton X-100. Cells were then incubated in block buffer without detergent for $30 \mathrm{~min}$. Coverslips were incubated with primary antibodies diluted in block buffer (without detergent) for $1 \mathrm{~h}$, washed multiple times with $1 \mathrm{x}$ PBS followed by incubation with secondary antibodies for $1 \mathrm{~h}$ at room temperature. Coverslips were washed again and mounted using Prolong Diamond Antifade mountant (Thermo Fisher). Images were acquired on a single z-plane on Lionheart ${ }^{\mathrm{TM}} \mathrm{FX}$ automated widefield microscope with a 20X Plan Fluorite WD 6.6 NP 0.45 objective. For Fig. 1e and Supplementary Fig. 1e, primary antibodies used: mouse anti-FLAG, M2 (1:500, Sigma-Aldrich, F1804) and rabbit anti-GM130, D6B1 (1:400, Cell Signaling Technologies, 12480). Secondary antibodies used: anti-mouse Alexa Fluor 647 (1:500, Invitrogen) and anti-rabbit Brilliant Violet 421 (1:100, Biolegend). YFP $(500 \mathrm{~nm})$, Texas Red $(590 \mathrm{~nm})$, and DAPI $(350 \mathrm{~nm})$ filter cubes were used to image Venus, FLAG, and GM130 respectively. For Fig. 2f: GFP $(465 \mathrm{~nm})$, Texas Red and DAPI filter cubes were used to image GFP, FLAG, and GM130, respectively.

Image analysis: Image analyses were performed in using $\mathrm{FIJI}^{70}$ and the EzColocalization ${ }^{71}$ plugin was used to determine the Pearson correlation coefficient for co-localization analyses in Fig. If and Supplementary Fig. 1f. For PM localization, a binary mask was generated from the thresholded Venus-RIT channel and saved as a selection (outer) to measure total signal intensity cell. The second mask was produced by five iterations of erosion function and subtracted from the outer mask using image calculator. The resulting mask (Supplementary Fig. 1d) was converted to a selection and used to measure the PM signal intensity.

\section{Detergent-assisted subcellular fractionation. COS-7 cells were seeded onto} $60 \mathrm{~mm}$ plates and transfected with FLAG-CNA $\beta 2$, FLAG-CNA $\beta 1$ (WT or C483S, C493S, C483/C493S) or EFR3B-FLAG at $80 \%$ confluency. In all, $48 \mathrm{~h}$ post transfection, cells were rinsed, harvested, and pellets snap-frozen in liquid nitrogen. Pellets were resuspended in $200 \mu$ digitonin buffer ( $10 \mathrm{mM}$ HEPES $\mathrm{pH} 6.8$, $100 \mathrm{mM} \mathrm{NaCl}, 300 \mathrm{mM}$ sucrose, $3 \mathrm{mM} \mathrm{MgCl}_{2}, 5 \mathrm{mM}$ EDTA and $0.015 \%$ Digitonin) supplemented with protease inhibitors by pipetting and rotating at $4{ }^{\circ} \mathrm{C}$ for $15 \mathrm{~min}$. Input (6\%) was taken as input prior to centrifugation at $2000 \times g$ for $20 \mathrm{~min}$. The supernatant was removed, centrifuged at $16,000 \times g$ for $5 \mathrm{~min}$ to remove any contamination from the pellet fraction, and saved as the cytosol fraction. The pellet was washed twice with ice-cold PBS and resuspended in $200 \mu \mathrm{l}$ Triton X-100 buffer (HEPES pH 7.5, $100 \mathrm{mM} \mathrm{NaCl}, 300 \mathrm{mM}$ sucrose, $3 \mathrm{mM} \mathrm{MgCl}$, $3 \mathrm{mM}$ EDTA, $1 \%$ Triton X-100) supplemented with protease inhibitors. Pellets were lysed for $30 \mathrm{~min}$ by rotating at $4{ }^{\circ} \mathrm{C}$ followed by centrifugation at $7000 \times g$ for $10 \mathrm{~min}$ and supernatant collected as the membrane fraction. The clarified supernatant is saved as the cytosol fraction. Inputs and equal volumes $(6 \%)$ of the cytosol and membrane fractions were mixed with 6X SDS sample buffer, heated to $95^{\circ} \mathrm{C}$ for $5 \mathrm{~min}$ and resolved by SDS-PAGE followed by immunoblotting. Primary antibodies used: anti-FLAG (1:2500; Sigma F3165), rabbit anti-calnexin (1:3000 ADI-SPA-865, Enzo Life Sciences), and anti-Gapdh (1:20,000, 1E6D9, Proteintech). After incubation with secondary antibodies: IRDye 680RD Goat anti-mouse IgG $(\mathrm{H}+\mathrm{L})(1: 15,000$, LiCOR Biosciences 926-68071) and IRDye 800CW Goat anti-rabbit IgG $(\mathrm{H}+\mathrm{L})$ (1:15,000, Li-COR Biosciences 926-32211), blots were imaged with the Li-Cor Odyssey imaging system. Enrichment in cytosol fraction was quantified as FLAG signal/Gapdh signal in cytosol fraction normalized to FLAG signal/Gapdh signal in inputs. Similarly for membrane enrichment, FLAG signal/Calnexin signal in membrane fraction normalized to FLAG signal/Calnexin signal in input. Statistical analysis was performed using GraphPad. Uncropped and unprocessed scans of the blots are provided in the Source Data file.

Acyl-resin assisted capture. The Acyl-RAC protocol was performed as described previously ${ }^{34}$ with minor changes. In brief, COS-7 cells were seeded on $60 \mathrm{~mm}$ plates and transfected at 70\% confluency with FLAG-CNA $\beta 2$, FLAG-CNA $\beta 1$ (WT or C483S, C493S, C483/C493S) or EFR3B-FLAG using JetOptimus. In all, $48 \mathrm{~h}$ following transfection, cells were harvested in ice-cold PBS and snap frozen in liquid nitrogen. Pellets were lysed in TAE lysis buffer (50 mM TEA pH 7.3, $150 \mathrm{mM} \mathrm{NaCl}, 2.5 \%$ SDS) supplemented with $1 \mathrm{mM}$ PMSF and protease inhibitors, vortexed briefly and incubated at $37^{\circ} \mathrm{C}$ for $20 \mathrm{~min}$ with constant gentle agitation. Lysates were subjected to fine needle aspiration with sterile 27.5-gauge needle and clarified by centrifugation $(16,000 \times g$ for $20 \mathrm{~min})$. In all, $400 \mu \mathrm{g}$ of each lysate was diluted to $2 \mathrm{mg} / \mathrm{ml}$ with lysis buffer and incubated with $10 \mathrm{mM}$ TCEP (646547, Sigma-Aldrich) for $30 \mathrm{~min}$, nutating at room temperature. In total, $25 \mathrm{mM} \mathrm{NEM}$ ( $\mathrm{N}$-ethylmaleimide, 40526, Alfa Aesar) was then added to the mix and incubated by gentle mixing at $40^{\circ} \mathrm{C}$ for $2 \mathrm{~h}$ to block free thiols. NEM was removed by acetone precipitation by adding four volumes of ice-cold acetone. Proteins were allowed to precipitate at $-20^{\circ} \mathrm{C}$ overnight. Following centrifugation of the solution at $16,000 \times g$ for $15 \mathrm{~min}$, the pellets were extensively washed with $70 \%$ acetone and the pellets were airdried for $5 \mathrm{~min}$ at room temperature. Pellets were resuspended in $200 \mu \mathrm{l}$ of binding buffer (50 mM TEA pH 7.3, $150 \mathrm{mM} \mathrm{NaCl}, 1 \mathrm{mM}$ EDTA, $1 \%$ SDS. $0.2 \%$ Triton X-100) by heating at $40{ }^{\circ} \mathrm{C}$ with frequent mixing. Approximately $20 \mu \mathrm{l}$ from each sample was taken as input and the rest were split into two $1.5-\mathrm{ml}$ microcentrifuge tubes. To capture S-palmitoylated proteins, $40 \mu \mathrm{l}$ prewashed thiopropyl Sepharose 6b (T8387, Sigma-Aldrich, prepared fresh) was added to samples in the presence of either $0.75 \mathrm{M} \mathrm{NH}_{2} \mathrm{OH}$ (from $2.5 \mathrm{M}$ stock, $\mathrm{pH} 7.5$, freshly diluted from Hydroxylamine solution (467804, Sigma-Aldrich)) or binding buffer (without SDS and EDTA for the negative control). Binding reactions were carried out on a rotator at $30^{\circ} \mathrm{C}$ for $4 \mathrm{~h}$. Resins were washed $4-5 \mathrm{x}$ with binding buffer, $5 \mathrm{~min}$ each, and proteins were eluted in $30 \mu \mathrm{l}$ binding buffer supplemented with $50 \mathrm{mM}$ DTT shaking at $30^{\circ} \mathrm{C}$ for $30 \mathrm{~min}$. In all, 6x SDS sample buffer was added to the samples followed by heating to $95^{\circ} \mathrm{C}$ for $5 \mathrm{~min}$. Inputs and eluates were separated by SDS-PAGE and transferred to nitrocellulose for western blotting with mouse anti- FLAG (1:2500; Sigma F3165) and rabbit anti-calnexin (1:3000 ADISPA-865, Enzo Life Sciences) antibodies. After incubation with secondary Li-Cor antibodies, blots were imaged with the Li-Cor Odyssey imaging system. Uncropped and unprocessed scans of the blots are provided in the Source Data file.

Acyl-PEG exchange. The previously published protocol ${ }^{35}$ was modified as follows: COS-7 cells expressing the indicated proteins were lysed and subjected to reductive alkylation with TCEP and NEM as described in the Acyl-RAC protocol. Following alkylation of total lysate $(300-400 \mu \mathrm{g})$ proteins were precipitated with four volumes of ice-cold Acetone at $-20^{\circ} \mathrm{C}$ overnight. Pellets were washed extensively with $70 \%$ Acetone, air dried for $5 \mathrm{~min}$ and resuspended in $72 \mu \mathrm{l}$ TEA buffer $\mathrm{pH} 7.3$, with $4 \%$ SDS ( $50 \mathrm{mM}$ TEA, $150 \mathrm{mM} \mathrm{NaCl}, 0.2 \%$ Triton X-100, $4 \mathrm{mM}$ EDTA) by heating to $40^{\circ} \mathrm{C}$ for an hour with constant mixing. Lysate was clarified by centrifugation at $16,000 \times g$ for $5 \mathrm{~min}$. Approximately $7 \mu \mathrm{l}(10 \%)$ from each sample was removed as input, the rest was split into two $30 \mu \mathrm{l}$ aliquots. For $\mathrm{NH}_{2} \mathrm{OH}$ treated sample, $36 \mu \mathrm{l}$ $\mathrm{NH}_{2} \mathrm{OH}(2.5 \mathrm{M}$ stock) was added and brought up to $120 \mu \mathrm{l}$ with TEA buffer with $0.2 \%$ Triton X-100 (50 mM TEA, $150 \mathrm{mM} \mathrm{NaCl})$. For negative control not treated with $\mathrm{NH}_{2} \mathrm{OH}, 90 \mu \mathrm{l}$ TEA buffer with $0.2 \%$ Triton X-100 was added. After incubation at $30^{\circ} \mathrm{C}$ for $1 \mathrm{~h}$ on a rotator, proteins were precipitated using methanolchloroform- $\mathrm{H}_{2} \mathrm{O}$, briefly air dried and resuspended in $30 \mu \mathrm{l}$ TEA buffer with $4 \%$ SDS, $50 \mathrm{mM}$ TEA, $150 \mathrm{mM} \mathrm{NACl}, 0.2 \%$ Triton X-100, $4 \mathrm{mM}$ EDTA by gentle mixing at $40{ }^{\circ} \mathrm{C}$. Each sample was treated with $90 \mu \mathrm{l}$ TEA buffer with $1.33 \mathrm{mM}$ mPEG-Mal (Methoxypolyethylene glycol maleimide, $5 \mathrm{kDa}, 63187$ Sigma-Aldrich) for a final concentration of $1 \mathrm{mM}$ mPEG-Mal. Samples were incubated for $2 \mathrm{~h}$ at RT with agitation before a final methanol-chloroform $-\mathrm{H}_{2} \mathrm{O}$ precipitation. The pellets were resuspended in $50 \mu \mathrm{l}$ TAE lysis buffer $(50 \mathrm{mM}$ TEA pH 7.3, $150 \mathrm{mM}$ $\mathrm{NaCl}, 2.5 \% \mathrm{SDS}$ ) and $10 \mu \mathrm{l}$ 6X SDS sample buffer was added before heating the sampled for $5 \mathrm{~min}$ at $95^{\circ} \mathrm{C}$. Typically $14 \mu \mathrm{l}$ of each sample was separated by SDS-PAGE and analyzed by immunoblot with FLAG and Calnexin antibodies. After incubation with secondary Li-Cor antibodies, blots were imaged with the LiCor Odyssey imaging system. Uncropped and unprocessed scans of the blots are provided in the Source Data file.

Pulse-chase metabolic labeling with 17-ODYA and L-AHA. COS-7 cells were transfected with cDNA encoding FLAG-CNA $\beta 1$ using Lipofectamine 2000 as per manufacturer's instructions. Twenty hours following transfection, cells were washed in phosphate-buffered saline (PBS) and starved in methionine-free DMEM containing 5\% charcoal-filtered FBS (Life Technologies), supplemented with $1 \mathrm{mM}$ L-glutamine and $1 \mathrm{mM}$ sodium pyruvate for $1 \mathrm{~h}$. Cells were then briefly washed in PBS then labeled with $30 \mu \mathrm{M}$ 17-ODYA and $50 \mu \mathrm{M}$ L-AHA for $2 \mathrm{~h}$ in this media. Labeling media was removed, cells were washed twice in PBS before chasing in complete DMEM supplemented with $10 \%$ FBS and $300 \mu \mathrm{M}$ palmitic acid. Palmostatin B (Palm B) or DMSO (vehicle) were added at chase time 0 and Palm B was replaced every hour. At indicated time points, cells were washed twice in PBS and frozen at $-80^{\circ} \mathrm{C}$ until processing. Cells were lysed with $500 \mu \mathrm{l}$ triethanolamine (TEA) lysis buffer (1\% Triton X-100, $150 \mathrm{mM} \mathrm{NaCl}, 50 \mathrm{mM}$ TEA pH 7.4, 100xEDTA-free Halt Protease Inhibitor [Life Technologies]). The lysates were transferred to $1.5-\mathrm{ml}$ Eppendorf tubes (Corning), vigorously shaken while placed on ice in between each agitation. Lysates were cleared by centrifugation at $13,000 \times g$ for $15 \mathrm{~min}$ at $4{ }^{\circ} \mathrm{C}$. Solubilized proteins in the supernatant were quantified using Bicinchoninic acid (BCA) assay (Life Technologies), $650 \mu \mathrm{g}-1 \mathrm{mg}$ of the lysate was added to Protein A-Sepharose beads (GE Healthcare) pre-incubated for 3-7 h with rabbit anti-FLAG antibody (Sigma-Aldrich) at $4{ }^{\circ} \mathrm{C}$. Immunoprecipitations were carried out overnight rotating at $4^{\circ} \mathrm{C}$.

Sequential on-bead CuAAC/click chemistry. Sequential on-bead click chemistry of immunoprecipitated 17-ODYA/L-AHA-labeled proteins was carried out as previously described ${ }^{39}$ with minor modifications. After immunoprecipitation, Sepharose beads were washed thrice in RIPA buffer, and on-bead conjugation of 
AF647 to 17-ODYA was carried out for $1 \mathrm{~h}$ at room temperature in $50 \mu \mathrm{l}$ of freshly mixed click chemistry reaction mixture containing $1 \mathrm{mM}$ TCEP, $1 \mathrm{mM}$

$\mathrm{CuSO}_{4} .5 \mathrm{H}_{2} \mathrm{O}, 100 \mu \mathrm{M}$ TBTA, and $100 \mathrm{mM}$ AF647-azide in PBS. After three washes in $500 \mu \mathrm{l}$ ice-cold RIPA buffer, conjugation of AF488 to L-AHA was carried out for $1 \mathrm{~h}$ at room temperature in $50 \mu \mathrm{l}$ click-chemistry reaction mixture containing $1 \mathrm{mM}$ TCEP, $1 \mathrm{mM}$ CuSO4.5H2O, $100 \mu \mathrm{M}$ TBTA, and $100 \mathrm{mM}$ AF488-alkyne in RIPA buffer. Beads were washed thrice with RIPA buffer and resuspended in $10 \mu \mathrm{l}$ SDS buffer ( $150 \mathrm{mM} \mathrm{NaCl}, 4 \%$ SDS, $50 \mathrm{mM}$ TEA pH 7.4), $4.35 \mu \mathrm{l} 4 \mathrm{X}$ SDS-sample buffer (8\% SDS, $4 \%$ Bromophenol Blue, $200 \mathrm{mM}$ Tris-HCl pH 6.8, 40\% Glycerol), and $0.65 \mu \mathrm{l} \mathrm{2-mercaptoethanol.} \mathrm{Samples} \mathrm{were} \mathrm{heated} \mathrm{for} 5 \mathrm{~min}$ at $90^{\circ} \mathrm{C}$ and separated on $10 \%$ tris-glycine SDS-PAGE gels for subsequent in-gel fluorescence analyses. A Typhoon Trio scanner (GE Healthcare) was used to measure in-gel fluorescence of SDS-PAGE gels: AF488 signals were acquired using the blue laser (excitation $488 \mathrm{~nm}$ ) with a 520BP40 emission filter, AF647 signals were acquired using the red laser (excitation $633 \mathrm{~nm}$ ) with a 670BP30 emission filter. Signals were acquired in the linear range and quantified using the ImageQuant TL7.0 software (GE Healthcare). For pulse-chase analyses, the ratio of palmitoylated substrates: total newly synthesized substrates were calculated as AF647/AF488 values at each time point, normalized to the value at $T=0$. Uncropped and unprocessed scans of the blots are provided in the Source Data file.

\section{Determination of palmitate incorporation in the presence of thioesterases.} COS-7 cells were seeded onto $60 \mathrm{~mm}$ plates and transfected with GFP- CNA $\beta 1$ together with vector, ABHD17A-FLAG (WT or S190A mutant), FLAG-APT2 or mCherry-APT1. In all, $24 \mathrm{~h}$ post-transfection, media was replaced with DMEM containing $2 \%$ FBS and labelled with $30 \mu \mathrm{M}$ 17-ODYA (17-Octadecynoic Acid,34450, Cayman Chemicals) or DMSO for $3 \mathrm{~h}$ at $37^{\circ} \mathrm{C}$ incubator. Cells for rinsed thrice with ice-cold PBS, harvested, and pellets were snap-frozen in liquid nitrogen. Pellets were then lysed in TEA lysis buffer (50 mM TEA pH 7.4, $150 \mathrm{mM}$ $\mathrm{NaCl}, 1 \%$ Triton X-100, $1 \mathrm{mM}$ PMSF) supplemented with protease inhibitors by rotating for $20 \mathrm{~min}$ at $4{ }^{\circ} \mathrm{C}$. Lysates were subjected to fine-needle aspiration with a sterile $27 \mathrm{G}$ syringe and clarified by centrifugation at $16,000 \times \mathrm{g}$ for $15 \mathrm{~min}$. In total, $300-400 \mu \mathrm{g}$ of each lysate was adjusted to $1 \mathrm{mg} / \mathrm{ml}$ with TAE lysis buffer and bound to $10 \mu \mathrm{l}$ pre-washed GFP-trap magnetic particles in for $1-2 \mathrm{~h}$ rotating end-over-end at $4{ }^{\circ} \mathrm{C}$. Input $(5 \%)$ was taken prior to bead binding. Beads were washed thrice in modified RIPA buffer (50 mM TAE pH 7.4, $150 \mathrm{mM} \mathrm{NaCl}, 1 \%$ Triton X-100, $1 \%$ sodium deoxycholate, $0.1 \%$ SDS). Proteins bound to beads were conjugated to azide-biotin in $50 \mu \mathrm{l}$ PBS with click chemistry reactants for $1 \mathrm{~h}$ at RT with constant agitation. Click chemistry reactants were freshly prepared as a $5 \mathrm{X}$ master mix that consists of $0.5 \mathrm{M}$ biotin-azide (Biotin-Picolyl azide, 1167, Click Chemistry Tools), $5 \mathrm{mM}$ TCEP, $0.5 \mathrm{mM}$ TBTA (Tris[(1-benzyl-1H-1,2,3-Triazol-4-yl)methyl]amine, Sigma-Aldrich), and $5 \mathrm{mM} \mathrm{CuSO}_{4} \cdot 5 \mathrm{H}_{2} \mathrm{O}$. Beads were washed thrice in modified RIPA buffer and proteins eluted by boiling in 2X SDS sample buffer before resolving with SDS-PAGE. Anti-GFP (1:4,000, Living Colors, 632380, Clontech) was used to probe for GFP-CNA $\beta 1$, biotin incorporation was detected using fluorophore conjugated Streptavidin antibody (Licor IRDye 800CW Steptavidin, LI-COR Biosciences). ABHD17A and APT2 levels were determined using antiFLAG $(1: 2,500 ;$ F3165, Sigma- Aldrich) and APT1 was detected using for anti-RFP $(1: 3000 ; 22904$, Rockland Inc.). Level of GFP-CNA $\beta 1$ palmitoylation was quantified as streptavidin signal normalized to bound GFP signal. Statistical analyses were performed in GraphPad. Uncropped and unprocessed scans of the blots are provided in the Source Data file.

\section{Affinity purification coupled to mass spectrometry analyses}

Protein expression and FLAG Affinity purification. Flp-In T-REx 293 cells at $\sim 60-70 \%$ confluence were induced with $1 \mu \mathrm{g} / \mathrm{ml}$ tetracycline for $24 \mathrm{~h}$. Subconfluent cells ( $\sim 85-95 \%$ confluent) were harvested as follows: medium was drained from the plate, $0.5 \mathrm{ml}$ ice-cold PBS was added, and the cells were scraped (using a silicon cake spatula) and transferred to a $1.5 \mathrm{ml}$ Eppendorf tube on ice. Cells were collected by centrifugation $\left(5 \mathrm{~min}, 1500 \times g, 4^{\circ} \mathrm{C}\right)$, the PBS aspirated, and cells resuspended in $1 \mathrm{ml}$ ice-cold PBS prior to centrifugation $\left(5 \mathrm{~min}, 1500 \times \mathrm{g}, 4^{\circ} \mathrm{C}\right)$. This step was repeated once more, the remaining PBS was aspirated, and the weight of the cell pellet was determined. Cell pellets were frozen on dry ice and transferred to $-80^{\circ} \mathrm{C}$ until processing.

Affinity purification. Cells were lysed by passive lysis assisted by freeze-thaw. Briefly, to the frozen cell pellet, a 1:4 pellet weight:volume ratio of ice-cold lysis buffer was added, and the frozen pellet was resuspended by pipetting up and down. The lysis buffer was $50 \mathrm{mM}$ HEPES- $\mathrm{NaOH} \mathrm{pH} 8.0,100 \mathrm{mM} \mathrm{KCl}, 2 \mathrm{mM}$ EDTA, $0.1 \%$ NP40, $10 \%$ glycerol, $1 \mathrm{mM}$ PMSF, $1 \mathrm{mM}$ DTT and Sigma protease inhibitor cocktail, P8340, 1:500. Tubes were frozen and thawed once by placing on dry ice for 5-10 min, then incubated in a $37^{\circ} \mathrm{C}$ water bath with agitation until only a small amount of ice remained. Thawed samples were then put on ice, and the lysate transferred to $2 \mathrm{ml}$ Eppendorf tubes. An aliquot $(20 \mu \mathrm{l})$ was taken to monitor solubility. This aliquot was spun down, the supernatant transferred to a fresh tube, and $6 \mu \mathrm{l} 4 \mathrm{X}$ Laemmli sample buffer added. The pellet was resuspended in $26 \mu \mathrm{l} 2 \mathrm{X}$ Laemmli sample buffer). The $2 \mathrm{ml}$ tubes were centrifuged at $14,000 \mathrm{rpm}$ for $20 \mathrm{~min}$ at $4{ }^{\circ} \mathrm{C}$, and the supernatant transferred to fresh $15 \mathrm{ml}$ conical tubes. During centrifugation, anti-FLAG M2 magnetic beads (SIGMA) were prepared: $25 \mu \mathrm{l} 50 \%$ slurry was aliquoted for each IP (two $150 \mathrm{~mm}$ plates), and the beads were washed in batch mode with $3 \times 1 \mathrm{ml}$ of lysis buffer. To the rest of the lysate, the equivalen of $12.5 \mu \mathrm{l}$ packed FLAG M2 magnetic beads was added, and the mixture incubated $2 \mathrm{~h}$ at $4{ }^{\circ} \mathrm{C}$ with gentle agitation (nutator). Beads were pelleted by centrifugation $(1000 \mathrm{rpm}$ for $1 \mathrm{~min}$ ) and a $15 \mu \mathrm{l}$ aliquot of the lysate post-IP was taken for analysis. Most of the supernatant was removed with a pipette, and the beads were transferred with $\sim 200 \mu \mathrm{l}$ of lysis buffer to a fresh $1.7 \mathrm{ml}$ Eppendorf tube, magnetized for $\sim 30 \mathrm{~s}$, and the remaining buffer was aspirated. Two washes with $1 \mathrm{ml}$ lysis buffer and two washes with $20 \mathrm{mM}$ Tris- $\mathrm{HCl}\left(\mathrm{pH}\right.$ 8.0) $2 \mathrm{mM} \mathrm{CaCl}_{2}$ were performed. Briefly, for each of these quick washes, the sample was demagnetized, resuspended by pipetting up and down in the wash buffer, remagnetized for $\sim 30 \mathrm{sec}$, and the supernatant aspirated (a complete wash cycle takes between 1-2 min). After the last wash, most of the liquid was removed, the tube was spun briefly (1000 rpm for $1 \mathrm{~min}$ ).

Tryptic digestion. Following affinity purification, the beads were resuspended in $5 \mu$ of $20 \mathrm{mM}$ Tris- $\mathrm{HCl}$ (pH 8.0). $500 \mathrm{ng}$ of trypsin (Sigma Trypsin Singles, T7575; resuspended at $200 \mathrm{ng} / \mathrm{ul}$ in Tris buffer) was added, and the mixture was incubated at $37^{\circ} \mathrm{C}$ with agitation for $4 \mathrm{hr}$. After this first incubation, the sample was magnetized and the supernatant transferred to a fresh tube. Another $500 \mathrm{ng}$ of trypsin was added, and the resulting sample was incubated at $37^{\circ} \mathrm{C}$ overnight (no agitation required). The next morning, formic acid was added to the sample to a final concentration of $2 \%$ (from a $50 \%$ stock solution).

Mass spectrometry. Half the sample was used per analysis. A spray tip was formed on fused silica capillary column $(0.75 \mu \mathrm{m}$ ID, $350 \mu \mathrm{m}$ OD) using a laser puller (program $=4$; heat $=280, \mathrm{FIL}=0, \mathrm{VEL}=18, \mathrm{DEL}=200)$. In all, $10 \mathrm{~cm}( \pm 1 \mathrm{~cm})$ of C18 reversed-phase material (Reprosil-Pur $120 \mathrm{C} 18-\mathrm{AQ}, 3 \mu \mathrm{m}$ ) was packed in the column by pressure bomb (in $\mathrm{MeOH}$ ). The column was then pre-equilibrated in buffer A $(6 \mu \mathrm{l})$ before being connected in-line to a NanoLC-Ultra 2D plus HPLC system (Eksigent, Dublin, USA) coupled to an LTQ-Orbitrap Velos (Thermo Electron, Bremen, Germany) equipped with a nanoelectrospray ion source (Proxeon Biosystems, Odense, Denmark). The LTQ-Orbitrap Velos instrument under Xcalibur 2.0 was operated in the data-dependent mode to automatically switch between MS and up to 10 subsequent MS/MS acquisitions. Buffer A was $100 \% \mathrm{H}_{2} \mathrm{O}, 0.1 \%$ formic acid; buffer B was $100 \mathrm{ACN}, 0.1 \%$ formic acid. The HPLC gradient program delivered the acetonitrile gradient over $125 \mathrm{~min}$. For the first $20 \mathrm{~min}$, the flow rate was of $400 \mu \mathrm{l} / \mathrm{min}$ at $2 \% \mathrm{~B}$. The flow rate was then reduced to $200 \mu \mathrm{l} / \mathrm{min}$ and the fraction of solvent B increased in a linear fashion to $35 \%$ until min 95.5. Solvent B was then increased to $80 \%$ over $5 \mathrm{~min}$ and maintained at that level until $107 \mathrm{~min}$. The mobile phase was then reduced to $2 \% \mathrm{~B}$ until the end of the run $(125 \mathrm{~min})$. The parameters for data-dependent acquisition on the mass spectrometer were: 1 centroid MS (mass range 400-2000) followed by MS/MS on the 10 most abundant ions. General parameters were: activation type $=$ CID, isolation width $=1 \mathrm{~m} / \mathrm{z}$, normalized collision energy $=35$, activation $\mathrm{Q}=0.25$, activation time $=10 \mathrm{~ms}$. For data-dependent acquisition, minimum threshold was 500 , the repeat count $=1$, repeat duration $=30 \mathrm{~s}$, exclusion size list $=500$, exclusion duration $=30 \mathrm{~s}$, exclusion mass width (by mass) $=$ low 0.03 , high 0.03 .

Mass spectrometry data extraction. RAW mass spectrometry files were converted to mzXML using ProteoWizard (3.0.4468) and analyzed using the iProphet pipeline ${ }^{72}$ implemented within ProHits ${ }^{73}$ as follows. The database consisted of the human and adenovirus complements of the RefSeq protein database (version 57) supplemented with "common contaminants" from the Max Planck Institute (http:// lotus1.gwdg.de/mpg/mmbc/maxquant_input.nsf/7994124a4298328fc125748d0048 fee2/\$FILE/contaminants.fasta) and the Global Proteome Machine (GPM; http:// www.thegpm.org/crap/index.html). The search database consisted of forward and reversed sequences (labeled "DECOY"); in total 72,226 entries were searched. The search engines used were Mascot (2.3.02; Matrix Science) and Comet ${ }^{74}$ (2012.01 rev.3) with trypsin specificity (two missed cleavages were allowed) and deamidation (NQ) and oxidation (M) as variable modifications. Charges of $+2,+3$, and +4 were allowed, and the parent mass tolerance was set at $12 \mathrm{ppm}$ while the fragment bin tolerance was set at $0.6 \mathrm{amu}$. The resulting Comet and Mascot search results were individually processed by PeptideProphet ${ }^{75}$ and peptides were assembled into proteins using parsimony rules first described in ProteinProphet ${ }^{76}$ into a final iProphet protein output using the Trans-Proteomic Pipeline (TPP; Linux version, v0.0 Development trunk rev 0, Build 201303061711). TPP options were as follows: general options were -p0.05-x20 -PPM -d"DECOY", iProphet options were -ipPRIME and PeptideProphet options were-OpdP. All proteins with a minimal iProphet protein probability of 0.05 were parsed to the relational module of ProHits. Note that for analysis with SAINT, only proteins with iProphet protein probability $\geq 0.95$ are considered. This corresponds to an estimated protein-level FDR of $\sim 5 \%$. Statistical analysis was performed with SAINTexpress (with default parameters), using 38 biological replicates of FLAG-GFP (all from asynchronous HEK293 T-REx cells, all run on the Orbitrap Velos) as negative controls, including two samples run in tandem with the two biological replicates. Full list of highconfidence interaction partners are provided in Supplementary Data 1. The AP-MS data generated in this study have been deposited to the ProteomeXchange database through partner MassIVE under accession codes PXD026809 and MSV000087664, respectively (see Data Availability). 


\section{In vitro peptide- calcineurin-binding assays}

Purification of calcineurin. 6xHis-tagged human calcineurin A ( $\alpha$ isoform, truncated at residue 392 ), WT or ${ }^{330} \mathrm{NIR}^{332}$-AAA mutant were expressed in tandem with the calcineurin B subunit in E. coli BL21 (DE3) cells (Invitrogen, USA) and cultured in LB medium containing carbenicillin $(50 \mu \mathrm{g} / \mathrm{ml})$ at $37^{\circ} \mathrm{C}$ to mid-log phase. Expression was induced with $1 \mathrm{mM}$ IPTG at $16^{\circ} \mathrm{C}$ for $18 \mathrm{~h}$. Cells were pelleted, washed, and frozen at $-80^{\circ} \mathrm{C}$ for at least $12 \mathrm{~h}$. Thawed cell pellets were resuspended in lysis buffer (50 mM Tris- $\mathrm{HCl}$ pH 7.5, $150 \mathrm{mM} \mathrm{NaCl}, 0.1 \%$ Tween 20, $1 \mathrm{mM} \beta$-mercaptoethanol, protease inhibitors) and lysed by sonication using four, 1 -minute pulses at $40 \%$ output. Extracts were clarified using two rounds of centrifugation $(20,000 \times \mathrm{g}, 20 \mathrm{~min})$ and then bound to $1 \mathrm{ml}$ of Ni-NTA agarose beads (Invitrogen) in lysis buffer containing $5 \mathrm{mM}$ imidazole for $2-4 \mathrm{~h}$. at $4{ }^{\circ} \mathrm{C}$, in batch. Bound beads were loaded onto a column and washed with lysis buffer containing $20 \mathrm{mM}$ imidazole and eluted with lysis buffer containing $300 \mathrm{mM}$ imidazole, $\mathrm{pH}$ 7.5. Purified calcineurin heterodimer were dialyzed in buffer $(50 \mathrm{mM}$ Tris- $\mathrm{HCl} \mathrm{pH}$ $7.5,150 \mathrm{mM} \mathrm{NaCl}, 1 \mathrm{mM} \beta$-mercaptoethanol) and stored in $10 \%$ glycerol at $-80^{\circ} \mathrm{C}$.

Peptide purification. In all, 16mer peptides were fused to GST in vector pGEX-4T-3 and expressed in E. coli BL21 (DE3) (Invitrogen). Cells were grown at $37^{\circ} \mathrm{C}$ to mid$\log$ phase and induced with $1 \mathrm{mM}$ IPTG for $2 \mathrm{~h}$. Cell lysates were prepared using the EasyLyse ${ }^{\mathrm{TM}}$ bacterial protein extract solution (Lucigen Corp. USA) or the CelLytic B reagent (Sigma, USA) according to the manufacturers' protocol and were stored at $-80^{\circ} \mathrm{C}$

In vitro binding. In all, 1-2 $\mu \mathrm{g}$ His-tagged calcineurin was first bound to magnetic Dynabeads (Thermo Fisher Sci. USA) in base buffer $(50 \mathrm{mM}$ Tris- $\mathrm{HCl} \mathrm{pH} 7.5$, $150 \mathrm{mM} \mathrm{NaCl}, 0.1 \%$ Tween $20,1 \mathrm{mM} \beta$-mercaptoethanol, protease inhibitors, 5-10 mM imidazole, $1 \mathrm{mg} / \mathrm{ml} \mathrm{BSA}$ ) for $1 \mathrm{~h}$ at $4{ }^{\circ} \mathrm{C}$. In all, $4-5 \mu \mathrm{g}$ GST-peptide were then added to the binding reaction and incubated further for $3 \mathrm{~h}$. In total, $3 \%$ of the reaction mix was removed as 'input' prior to the incubation, boiled in 2X-SDS sample buffer, and stored at $-20^{\circ} \mathrm{C}$. The beads were washed in base buffer con taining 15-20 mM imidazole and bound proteins were eluted by boiling in SDS sample buffer for $5 \mathrm{~min}$ followed by SDS-PAGE and immunoblotting with antiGST (BioLegend MMS-112P) and anti-His (Qiagen 34660) antibodies. Blots were imaged with the Li-Cor Odyssey imaging system. GST peptides co-purifying with HIS-CN were normalized to their respective input and amount of calcineurin pulled down. Co-purification with $\mathrm{CN}$ was reported relative to that of the peptide with the known PxIxIT motif from NFATC1: PALESPRIEITSCLGL. For (Supplementary Fig. 4B, FAM126A peptides used were FAM126A PSISIT:

SGQQRPPSISITLSTD and FAM126A ASASAA Mut: SGQQRPASASAALSTD. Statistical significance was determined with unpaired Student's $t$ test, using GraphPad. Uncropped and unprocessed scans of the blots are provided in the Source Data file.

Immunoprecipitations. Cells expressing indicated plasmids were rinsed with icecold PBS, harvested and pellets snap-frozen in liquid nitrogen and stored at $-80^{\circ} \mathrm{C}$ until use. Cell pellets were lysed in lysis buffer (50 mM Tris, pH 7.5, $150 \mathrm{mM} \mathrm{NaCl}$, $1 \%$ Triton X-100) supplemented with a protease and phosphatase inhibitor cocktail (Halt ${ }^{\mathrm{TM}}$, ThermoFisher) and $250 \mathrm{U} / \mathrm{ml}$ benzonase for $30 \mathrm{~min}$ rotating end-overend at $4{ }^{\circ} \mathrm{C}$ and subjected to fine needle aspiration using a sterile 27.5-gauge needle. Cell lysates were clarified by centrifugation at $16,000 \times g$ for $20 \mathrm{~min}$ and protein concentrations determined by Bicinchoninic acid (BCA) assay (Life Technologies). In all, $600-1000 \mu \mathrm{g}$ of each lysate, adjusted to $1 \mathrm{mg} / \mathrm{ml}$ with binding buffer $(50 \mathrm{mM}$ Tris, pH 7.5, $150 \mathrm{mM} \mathrm{NaCl}, 0.5 \%$ Triton X-100) was incubated with Pierce antiHA (ThermoFisher) or GFP-Trap magnetic beads (Bulldog Bio. Inc.) and rotated for $2-4 \mathrm{~h}$ at $4{ }^{\circ} \mathrm{C}$. Beads were washed thrice in binding buffer and boiled in 2X SDS sample buffer for $5 \mathrm{~min}$. Input (2\%) and bound (100\%) fractions were resolved by SDS-PAGE and immunoblotted with HA ( $1: 2000$, H3663, Sigma-Aldrich), GFP ( $1: 4000$, Living Colors, 632380, Clontech), MYC (1:3000, 9B11, Cell Signaling Technologies), and $\beta$-Actin (1:3,000; 926-42210, Li-Cor Biosciences) antibodies followed by secondary Li-Cor antibodies. Blots were imaged with the Li-Cor Odyssey imaging system. Uncropped and unprocessed scans of the blots are provided in the Source Data file.

Proximity-dependent biotin identification (BiolD) analysis. HeLa cells were seeded onto $10 \mathrm{~cm}$ plates and transfected at $80 \%$ confluence with MYC-BirACNA $\beta 1$. In all, $24 \mathrm{~h}$ post transfection, cells were passaged onto two $10 \mathrm{~cm}$ plates. The next day, cells were co-transfected with EFR3B HA T2A TTC7B GFP P2A FLAG FAM126A (WT or ASASAA mutant) and HA-PI4KA. In all, $4 \mathrm{~h}$ posttransfection, media was replaced with fresh media containing $50 \mu \mathrm{M}$ D-biotin (Sigma B-4501). After $16 \mathrm{~h}$ of labeling, cells were collected and snap frozen in liquid nitrogen. Cells were lysed in RIPA buffer $(150 \mathrm{mM} \mathrm{NaCl}, 1 \%$ Triton X-100, $0.5 \%$ Deoxycholate, $0.1 \%$ SDS, $50 \mathrm{mM}$ Tris $\mathrm{pH} 8.0$ ) supplemented with a protease and phosphatase inhibitor cocktail (Halt ${ }^{\mathrm{TM}}$, ThermoFisher) and $250 \mathrm{U} / \mathrm{ml}$ benzonase (EMD Millipore) for $30 \mathrm{~min}$ rotating end-over-end at $4{ }^{\circ} \mathrm{C}$ and subjected to fine needle aspiration with a sterile 27.5 -gauge needle. Cell lysates were clarified by centrifugation $(16,000 \times g$ for $20 \mathrm{~min})$ and protein concentration determined with $\mathrm{BCA}$ analysis. For each binding reaction, $1 \mathrm{mg}$ of clarified lysate was incubated with
$30 \mu \mathrm{l}$ of pre-rinsed streptavidin magnetic particles (11641786001, Sigma-Aldrich) in $1 \mathrm{ml}$ RIPA buffer for $16 \mathrm{~h}$ with rotation at $4{ }^{\circ} \mathrm{C}$. An input aliquot $(20 \mu \mathrm{l})$ was removed prior to bead addition. Beads were washed thrice with $1 \mathrm{ml}$ RIPA buffer, rotating for $5 \mathrm{~min}$ each, and eluted in $2 \mathrm{X}$ sample buffer (10\%SDS, $0.06 \%$ Bromophenol blue, $50 \%$ glycerol, $0.6 \mathrm{M} \mathrm{DTT}, 375 \mathrm{mM}$ Tris- $\mathrm{HCl} \mathrm{pH} 6.8$ ). Inputs and bound $(100 \%)$ samples were boiled and resolved by SDS-PAGE followed by immunoblotting with mouse FLAG (1:2,500; F3165, Sigma- Aldrich), rabbit MYC (1:2,000; 71D10, Cell Signaling), mouse HA (1:2000, H3663, Sigma-Aldrich), mouse GFP (1:4,000, Living Colors, 632380, Clontech), and rabbit $\beta$-Actin ( 1 : 3000; 926-42210, Li-Cor Biosciences) antibodies. Blots were imaged with $\mathrm{Li}$ Cor Odyssey imaging system following incubation with secondary Li-Cor antibodies. Binding for each protein was quantified as their respective signals/MYC bound signal normalized to respective signals/Actin Input signal. Biotinylation of each protein in complex with WT FAM126A, is set to 1 . Statistical significance was determined using GraphPad.

\section{Hydrogen-deuterium exchange analysis (HDX-MS)}

Protein expression. GST-tagged human calcineurin A (residues 2-391 of human CNA alpha isoform) in tandem with calcineurin B subunit were expressed in BL21 C41 Escherichia coli, induced with $0.1 \mathrm{mM}$ IPTG (isopropyl $\beta$-d-1-thiogalactopyranoside) and grown at $23^{\circ} \mathrm{C}$ overnight. Cells were harvested, flash frozen in liquid nitrogen, and stored at $-80^{\circ} \mathrm{C}$ until use. Bacmids harboring MultiBac PI4KA complex constructs were transfected into Spodoptera frugiperda (Sf9) cells, and viral stocks amplified for one generation to acquire a P2 generation final viral stock. Final viral stocks were added to Sf9 cells at $\sim 1.8 \times 10^{6}$ in a $1 / 100$ to $1 / 50$ virus volume to cell volume ratio. Constructs were expressed for $68 \mathrm{~h}$ before pelleting of infected cells. Cell pellets were snap frozen in liquid nitrogen, followed by storage at $-80^{\circ} \mathrm{C}$.

Protein purification (GST tagged human calcineurin). Escherichia coli cell pellets were lysed by sonication for $5 \mathrm{~min}$ in lysis buffer [ $50 \mathrm{mM}$ Tris $\mathrm{pH} 8.0,100 \mathrm{mM}$ $\mathrm{NaCl}, 2 \mathrm{mM}$ EDTA, $2 \mathrm{mM}$ EGTA, protease inhibitors (Millipore Protease Inhibitor Cocktail Set III, Animal-Free)]. NaCl solution was added to $1 \mathrm{M}$ and the solution was centrifuged for $10 \mathrm{~min}$ at $12,000 \times g$ at $1{ }^{\circ} \mathrm{C}$ and for $20 \mathrm{~min}$ at $38,000 \times g$ at $1{ }^{\circ} \mathrm{C}$ (Beckman Coulter Avanti J-25I, JA 25.50 rotor). CHAPS was added to $0.02 \%$. Supernatant was loaded onto a $5 \mathrm{ml}$ GSTrap 4B column (GE) in a superloop for $45 \mathrm{~min}$ and the column was washed in Wash Buffer [50 mM Tris pH 8.0, $110 \mathrm{mM}$ KOAc, $2 \mathrm{mM} \mathrm{MgOAc,} 1 \mathrm{mM}$ DTT, 5\% glycerol (v/v), 0.02\% chaps] to remove nonspecifically bound proteins. The column was washed in Wash Buffer containing $2 \mathrm{mM}$ ATP to remove the GroEL chaperone. The GST-tag was cleaved by adding Wash Buffer containing PreScission protease to the column and incubating overnight at $4{ }^{\circ} \mathrm{C}$. Cleaved protein was eluted with Wash Buffer. Protein was concentrated using an Amicon $10 \mathrm{kDa}$ MWCO concentrator (MilliporeSigma) and size exclusion chromatography (SEC) was performed using a Superdex 75 10/300 column equilibrated in Wash Buffer. Fractions containing protein of interest were pooled, concentrated, flash frozen, and stored at $-80^{\circ} \mathrm{C}$.

Protein purification (PI4KA complex). Sf9 pellets were resuspended in lysis buffer $20 \mathrm{mM}$ imidazole $\mathrm{pH}$ 8.0, $100 \mathrm{mM} \mathrm{NaCl}, 5 \%$ glycerol, $2 \mathrm{mM} \beta \mathrm{Me}$, protease (Protease Inhibitor Cocktail Set III, Sigma)] and lysed by sonication. Triton X-100 was added to $0.1 \%$ final, and lysate was centrifuged for $45 \mathrm{~min}$ at $20,000 \times \mathrm{g}$ at $1{ }^{\circ} \mathrm{C}$ (Beckman Coulter Avanti J-25I, JA 25.50 rotor). Supernatant was loaded onto a HisTrap FF Crude column (GE Healthcare) and superlooped for $1 \mathrm{~h}$. The column was washed with Ni-NTA A buffer [20 mM imidazole pH 8.0, $100 \mathrm{mM} \mathrm{NaCl}, 5 \%$ glycerol $(\mathrm{v} / \mathrm{v}), 2 \mathrm{mM} \beta \mathrm{Me}$, washed with $6 \% \mathrm{Ni}-\mathrm{NTA} \mathrm{B}$ buffer $[30 \mathrm{mM}$ imidazole $\mathrm{pH} 8.0,100 \mathrm{mM} \mathrm{NaCl}, 5 \%(\mathrm{v} / \mathrm{v})$ glycerol, $2 \mathrm{mM} \beta \mathrm{Me}$ ), and the protein eluted with $100 \%$ Ni-NTA B buffer ( $450 \mathrm{mM}$ imidazole). Elution fractions were passed through a $5 \mathrm{ml}$ StrepTrapHP column pre-equilibrated in GF buffer [ $20 \mathrm{mM}$ imidazole $\mathrm{pH}$ $7.0,150 \mathrm{mM} \mathrm{NaCl}, 5 \%$ glycerol (v/v), $0.5 \mathrm{mM}$ TCEP]. The column was washed with GF buffer before loading a tobacco etch virus protease containing a stabilizing lipoyl domain (Lip-TEV), and cleavage proceeded overnight. Cleaved protein was eluted with GF buffer and concentrated down to $250 \mu \mathrm{l}$ in an Amicon $50 \mathrm{kDa}$ MWCO concentrator (MilliporeSigma) pre-equilibrated in GF buffer. Concentrated protein was flash frozen in liquid nitrogen and stored at $-80{ }^{\circ} \mathrm{C}$.

Mass spectrometry sample preparation. HDX reactions for PI4KA complex (PI4KIIIa, TTC7B, FAM126A) and Calcineurin were conducted in a final reaction volume of $24 \mu \mathrm{l}$ with a final concentration of $0.17 \mu \mathrm{M}$ ( 8 pmol) PI4KA complex and $0.95 \mu \mathrm{M}(24 \mathrm{pmol})$ Calcineurin. The reaction was initiated by the addition of $16 \mu$ of $\mathrm{D}_{2} \mathrm{O}$ buffer $\left(150 \mathrm{mM} \mathrm{NaCl}, 20 \mathrm{mM} \mathrm{pH} 8.0\right.$ Imidazole, $90 \% \mathrm{D}_{2} \mathrm{O}(\mathrm{V} / \mathrm{V})$ ) to $6.5 \mu$ of PI4KA or PI4K buffer solution and $0.66 \mu$ Calcineurin or Calcineurin buffer solution (final $\mathrm{D}_{2} \mathrm{O}$ concentration of $65 \%$ ). The reaction proceeded for $3,30,300$ or $3000 \mathrm{~s}$ at $20^{\circ} \mathrm{C}$, before being quenched with ice-cold acidic quench buffer, resulting in a final concentration of $0.6 \mathrm{M}$ guanidine- $\mathrm{HCl}$ and $0.9 \%$ formic acid post quench. All conditions and timepoints were generated in triplicate. Samples were flash frozen immediately after quenching and stored at $-80^{\circ} \mathrm{C}$ until injected onto the ultra-performance liquid chromatography (UPLC) system for proteolytic cleavage, peptide separation, and injection onto a QTOF for mass analysis, described below. 
Protein digestion and MS/MS data collection. Protein samples were rapidly thawed and injected onto an integrated fluidics system containing a HDx-3 PAL liquid handling robot and climate-controlled $\left(2^{\circ} \mathrm{C}\right)$ chromatography system (LEAP Technologies), a Dionex Ultimate 3000 UHPLC system, as well as an Impact HD QTOF Mass spectrometer (Bruker). The full details of the automated LC system are described $\mathrm{in}^{77}$. The protein was run over one immobilized pepsin column (Trajan; ProDx protease column, $2.1 \mathrm{~mm} \times 30 \mathrm{~mm}$ PDX.PP01-F32) at $200 \mu \mathrm{L} / \mathrm{min}$ for $3 \mathrm{~min}$ at $10^{\circ} \mathrm{C}$. The resulting peptides were collected and desalted on a C18 trap column (Acquity UPLC BEH C18 $1.7 \mathrm{~mm}$ column $(2.1 \times 5 \mathrm{~mm})$; Waters 186003975$)$. The trap was subsequently eluted in line with an ACQUITY $1.7 \mu \mathrm{m}$ particle,

$100 \times 1 \mathrm{~mm}^{2}$ C18 UPLC column (Waters), using a gradient of 3-35\% B (Buffer A $0.1 \%$ formic acid; Buffer B $100 \%$ acetonitrile) over 11 min immediately followed by a gradient of $35-80 \%$ over $5 \mathrm{~min}$. Mass spectrometry experiments acquired over a mass range from 150 to $2200 \mathrm{~m} / \mathrm{z}$ using an electrospray ionization source operated at a temperature of $200{ }^{\circ} \mathrm{C}$ and a spray voltage of $4.5 \mathrm{kV}$.

Peptide identification. Peptides were identified from the non-deuterated samples of PI4K using data-dependent acquisition following tandem MS/MS experiments $(0.5 \mathrm{~s}$ precursor scan from 150 to $2000 \mathrm{~m} / \mathrm{z} ; 120.25 \mathrm{~s}$ fragment scans from 150 to $2000 \mathrm{~m} /$ z). MS/MS datasets were analyzed using PEAKS7 (PEAKS), and peptide identification was carried out by using a false discovery-based approach, with a threshold set to $1 \%$ using a database of known contaminants found in Sf9 cells and BL21 C41 Escherichia coli $^{78}$. The search parameters were set with a precursor tolerance of $20 \mathrm{ppm}$, fragment mass error $0.02 \mathrm{Da}$, charge states from 1 to 8 , leading to a selection criterion of peptides that had a $-10 \log \mathrm{P}$ score of 35.4 and 29.3 for the PI4KA complex and calcineurin, respectively. A search for phosphorylated peptides was subsequently completed using PEAKS7 with a variable phosphorylation modification search with the same parameters as above, and a false discovery threshold of $0.1 \%$ using a database of known contaminants. The search parameters were otherwise the same as above leading to a selection criterion of peptides that had a $-10 \log \mathrm{P}$ score of 37.8 for the PI4KA complex. All putative phosphorylated peptides from the search were subjected to manual inspection, and the only one site (TTC7B S519) was identified. No phosphorylation of FAM126A was detected. The area of the phosphorylated and unphosphorylated peptides of TTC7 S159 were roughly equal, suggesting the purified trimer was $\sim 50 \%$ phosphorylated at this site.

Mass analysis of peptide centroids and measurement of deuterium incorporation. HD-Examiner Software (Sierra Analytics) was used to calculate the level of deuterium incorporation into each peptide. All peptides were manually inspected for correct charge state, correct retention time, and appropriate selection of isotopic distribution. Deuteration levels were calculated using the centroid of the experimental isotope clusters. Results are presented as relative levels of deuterium incorporation, with no correction for back exchange. The only correction was for the deuterium percentage of the buffer in the exchange reaction (65\%). Differences in exchange in a peptide were considered significant if they met all three of the following criteria: $\geq 5 \%$ change in exchange, $\geq 0.5 \mathrm{Da}$ difference in exchange, and a two-tailed T-test value of less than 0.01 . The raw HDX data are shown in two different formats. The raw peptide deuterium incorporation graphs for a selection of peptides with significant differences are shown in Fig. 4e, g, with the raw data for all analyzed peptides in the Source Data file. To allow for visualization of differences across all peptides, we utilized number of deuteron difference (\#D) plots (Fig. 4d). These plots show the total difference in deuterium incorporation over the entire H/D exchange time course, with each point indicating a single peptide. Samples were only compared within a single experiment and were never compared to experiments completed at a different time with a different final $\mathrm{D}_{2} \mathrm{O}$ level. The data analysis statistics for all HDX-MS experiments are in Supplementary Table 1 according to published guidelines ${ }^{79}$. The HDX-MS data generated in this study have been deposited to the ProteomeXchange database through partner PRIDE ${ }^{80}$ under accession code PXD025900.

In vivo analysis of FAM126A phosphorylation status. HeLa cells seeded onto $10 \mathrm{~cm}$ plates were transfected with EFR3B HA_T2A_TTC7B MYC_P2A_FLAG FAM126A WT or ASASAA mutant. In all, $24 \mathrm{~h}$ post-transfection, plates were washed, trypsinized, and passaged onto $60 \mathrm{~mm}$ plates for treatments. The next day, cells were pre-treated with $2 \mu \mathrm{M}$ FK506 (LC Laboratories) for $1 \mathrm{~h}, 2 \mu \mathrm{M}$ BIM (bisindolylmaleimide, Calbiochem) for $15 \mathrm{~min}$ or DMSO in growth media. Cells were then stimulated with $500 \mathrm{nM}$ PMA (Phorbol 12-myristate 13-acetate, SigmaAldrich) or DMSO for $15 \mathrm{~min}$, washed and harvested in ice-cold PBS. Pellets were snap-frozen in liquid nitrogen and stored at $-80{ }^{\circ} \mathrm{C}$ until use. Cells were lysed with RIPA buffer ( $150 \mathrm{mM} \mathrm{NaCl}, 1 \%$ Triton X-100, 0.5\% Deoxycholate, $0.1 \%$ SDS, $50 \mathrm{mM}$ Tris $\mathrm{pH}$ 8.0) supplemented with protease and phosphatase inhibitor cocktail (Halt ${ }^{\mathrm{TM}}$, ThermoFisher) and $250 \mathrm{U} / \mathrm{ml}$ benzonase for $30 \mathrm{~min}$ rotating endover-end at $4{ }^{\circ} \mathrm{C}$ and subjected to fine needle aspiration with a sterile 27.5-gauge needle. Cell lysates were clarified by centrifugation at $16,000 \times g$ for $20 \mathrm{~min}$ ) and protein concentration determines using BCA analysis. In all, $20 \mu \mathrm{g}$ of each lysate was analyzed by SDS-PAGE followed by immunoblotting with anti-FLAG $(1: 2500)$ and custom anti-phosphospecific FAM126A S485 (1:1000) antibodies. PKC activation was assessed by phosphorylation of the downstream substrate, ERK using p44/42 Erk1/2 antibody ( $1: 3000 ; 3 A 7$, Cell Signaling Technologies). Phosphorylation status of FAM126A in each treatment was quantified as pFAM126A
S485 signal/FLAG signal and reported relative to that in DMSO-treated FAM126A WT sample, using ImageStudio imaging software. Statistical significance was determined using GraphPad. Uncropped and unprocessed scans of the blots are provided in the Source Data file.

Bioluminescence resonance energy transfer measurements. HEK 293T cells were trypsinized and plated on white 96-well plates at a density of 75,000 cells/100 $\mu \mathrm{l}$ per well, together with the indicated DNA constructs $(0.15 \mu \mathrm{g}$ total DNA in $25 \mu \mathrm{l} \mathrm{per}$ well) and the cell transfection reagent (1.5 $\mu$ GeneCellin (Bulldog Bio) in $25 \mu \mathrm{l}$ per well) in Opti-MEM reduced serum medium (Gibco). Cells were transfected with DNA encoding the human M3 muscarinic receptor $(0.1 \mu \mathrm{g}$ total DNA/well) and the previously established SidM-2XP4M-based PI4P biosensor $^{22}$ ( $0.05 \mu \mathrm{g}$ total DNA/well). After $6 \mathrm{~h}$, media were replaced with $100 \mu \mathrm{l} /$ well of DMEM supplemented with $10 \%$ fetal bovine serum, $50 \mathrm{U} / \mathrm{ml}$ penicillin, and $50 \mu \mathrm{g} / \mathrm{ml}$ streptomycin. Measurements were performed $28 \mathrm{~h}$ post-transfection. Prior to measurements, media was replaced with $50 \mu \mathrm{l}$ buffer containing $120 \mathrm{mM} \mathrm{NaCl}, 4.7 \mathrm{mM} \mathrm{KCl}, 1.2 \mathrm{mM} \mathrm{CaCl}_{2}, 0.7 \mathrm{mM}$ $\mathrm{MgSO}_{4}, 10 \mathrm{mM}$ glucose, and $10 \mathrm{mM}$ Na-HEPES, $\mathrm{pH}$ 7.4. Cells were pretreated with FK506 $(1 \mu \mathrm{M})$, Cyclosporin A $(10 \mu \mathrm{M})$, or DMSO for $1 \mathrm{~h}$ at $37^{\circ} \mathrm{C}$. Measurements were performed at $37^{\circ} \mathrm{C}$ using a Varioskan Flash Reader (Thermo Scientific) and initiated with the addition of the cell permeable luciferase substrate, coelenterazine h $(20 \mu \mathrm{l}$, final concentration of $5 \mu \mathrm{M}$ ). Counts were recorded using 485 and $530 \mathrm{~nm}$ emission filters. Detection time was $250 \mathrm{~ms}$ for each wavelength. The indicated reagents were also dissolved in modified Krebs-Ringer buffer and were added manually in $10 \mu l$. For this, plates were unloaded, which resulted in an interruption in the recordings. All measurements were done at least in triplicate. BRET ratios were calculated by dividing the $530 \mathrm{~nm}$ and $485 \mathrm{~nm}$ intensities and results were normalized to the baseline. Since the absolute initial ratio values depended on the expression of the sensor, the resting levels were considered as $100 \%$, whereas the $0 \%$ was determined from values of those experiments where cytoplasmic Renilla luciferase construct was expressed alone.

Statistical analysis. Statistics were computed using Graphpad Prism 9. All data shown as representative images or as the mean of measurements with standard deviation (SD) error bars unless noted otherwise. All data represent at least three independent experiments and are indicated in each figure legend. For image analysis in Fig. If and Supplementary Fig. 1f, number of cells analyzed for GM130 co-localization from three independent experiments were as follows: $n=166$ for $\mathrm{CNA} \beta 2, n=164$ for CNA $\beta 1, n=130$ for $\mathrm{CNA} \beta 1^{\mathrm{C} 483}, n=128$ for $\mathrm{CNA} \beta 1^{\mathrm{C}}{ }^{493 \mathrm{~S}}$, $n=119$ for CNA $\beta 1^{\mathrm{C} 2 \mathrm{~S}}$. For plasma membrane signal ratio measurements: $n=75$ for CNA $\beta 2, n=86$ for CNA $\beta 1, n=98$ for CNA $\beta 1^{\mathrm{C} 483}, n=80$ for $\mathrm{CNA} \beta 1^{\mathrm{C}} 493 \mathrm{~S}$, $n=77$ for $\mathrm{CNA} \beta 1^{\mathrm{C} 2 \mathrm{~S}}$ from three independent experiments. Number of cells used for image analysis in Fig. $2 \mathrm{~g}$ from 4 independent experiments are $n=76$ for vector control, $n=94$ for wildtype ABHD17A, $n=89$ for ABHD17A S190A mutant. Figure $1 \mathrm{~g}$ immunoblot is representative of $n=5$ for EFR3B-FLAG, $n=5$ for FLAG-CNA $\beta 1, n=3$ for each CNA $\beta 1$ mutant (C483S, C493S, C2S), all replicates were independent experiments. Two-tailed unpaired Student's $t$-test was applied for statistical analyses between two groups. One-way analysis of variance (ANOVA) with appropriate multiple comparisons (all indicated in figure legends) were performed when comparing more than two groups.

Reporting summary. Further information on research design is available in the Nature Research Reporting Summary linked to this article.

\section{Data availability}

The data that support this study are available from the corresponding author upon reasonable request. The AP-MS data generated in this study have been deposited to the ProteomeXchange database through partner MassIVE under accession codes PXD026809 and MSV000087664, respectively. The HDX-MS data generated in this study have been deposited to the ProteomeXchange database through partner PRIDE under accession code PXD025900. Uncropped and unprocessed scans of blots and quantifications as well as HDX-MS source data are provided in the Source Data file. The imaging data that was used to generate Figs. $1 \mathrm{e}, \mathrm{f}$ and $2 \mathrm{f}$, g and Supplementary Fig. 1 e, $\mathrm{f}$ are published in Mendeley Data with DOI: $10.17632 / 85 \mathrm{v} 4 \mathrm{dj} 4 \mathrm{kgm} .1^{81}$. Structures of calcineurin heterodimer and the PI4KA trimer used in Fig. 4 are obtained from the Protein Data Bank with accession codes 6NUC and 6BQ1 respectively. Source data are provided with this article. Source data are provided with this paper.

Received: 25 June 2021; Accepted: 29 September 2021; Published online: 18 October 2021

\section{References}

1. Rusnak, F. \& Mertz, P. Calcineurin: form and function. Physiol. Rev. 80, 1483-1521 (2000)

2. Mansuy, I. M. Calcineurin in memory and bidirectional plasticity. Biochem. Biophys. Res. Commun. 311, 1195-1208 (2003). 
3. Schulz, R. A. \& Yutzey, K. E. Calcineurin signaling and NFAT activation in cardiovascular and skeletal muscle development. Dev. Biol. 266, 1-16 (2004).

4. Jain, J. et al. The T-cell transcription factor NFATp is a substrate for calcineurin and interacts with Fos and Jun. Nature 365, 352-355 (1993).

5. Liu, J. et al. Calcineurin is a common target of cyclophilin-cyclosporin A and FKBP-FK506 complexes. Cell 66, 807-815 (1991).

6. Wigington, C. P. et al. Systematic discovery of short linear motifs decodes calcineurin phosphatase signaling. Mol. Cell 79, 342-358 e312 (2020).

7. Brauer, B. L. et al. Leveraging new definitions of the LxVP SLiM to discover novel calcineurin regulators and substrates. ACS Chem. Biol. 14, 2672-2682 (2019).

8. Hashimoto, Y., Perrino, B. A. \& Soderling, T. R. Identification of an autoinhibitory domain in calcineurin. J. Biol. Chem. 265, 1924-1927 (1990).

9. Yang, S. A. \& Klee, C. B. Low affinity Ca2+-binding sites of calcineurin B mediate conformational changes in calcineurin A. Biochemistry 39, 16147-16154 (2000).

10. Guerini, D. \& Klee, C. B. Cloning of human calcineurin A: evidence for two isozymes and identification of a polyproline structural domain. Proc. Natl Acad. Sci. USA 86, 9183-9187 (1989).

11. Lara-Pezzi, E. et al. A naturally occurring calcineurin variant inhibits FoxO activity and enhances skeletal muscle regeneration. J. Cell Biol. 179, 1205-1218 (2007).

12. Bond, R., Ly, N. \& Cyert, M. S. The unique $\mathrm{C}$ terminus of the calcineurin isoform CNAbetal confers non-canonical regulation of enzyme activity by $\mathrm{Ca}(2+)$ and calmodulin. J. Biol. Chem. 292, 16709-16721 (2017).

13. Roy, J. \& Cyert, M. S. Identifying new substrates and functions for an old enzyme: Calcineurin. Cold Spring Harb. Perspect. Biol. 12, a035436 (2020).

14. Rodriguez, A. et al. A conserved docking surface on calcineurin mediates interaction with substrates and immunosuppressants. Mol. Cell 33, 616-626 (2009).

15. Grigoriu, S. et al. The molecular mechanism of substrate engagement and immunosuppressant inhibition of calcineurin. PLoS Biol. 11, e1001492 (2013).

16. Felkin, L. E. et al. Calcineurin splicing variant calcineurin Abeta1 improves cardiac function after myocardial infarction without inducing hypertrophy. Circulation 123, 2838-2847 (2011)

17. Lopez-Olaneta, M. M. et al. Induction of the calcineurin variant CnAbetal after myocardial infarction reduces post-infarction ventricular remodelling by promoting infarct vascularization. Cardiovasc. Res. 102, 396-406 (2014).

18. Padron-Barthe, L. et al. Activation of serine one-carbon metabolism by calcineurin Abeta1 reduces myocardial hypertrophy and improves ventricular function. J. Am. Coll. Cardiol. 71, 654-667 (2018).

19. Gomez-Salinero, J. M. et al. The calcineurin variant CnAbetal controls mouse embryonic stem cell differentiation by directing mTORC2 membrane localization and activation. Cell Chem. Biol. 23, 1372-1382 (2016).

20. Berridge, M. J. Inositol trisphosphate and diacylglycerol as second messengers. Biochem. J. 220, 345-360 (1984).

21. Balla, A. et al. Maintenance of hormone-sensitive phosphoinositide pools in the plasma membrane requires phosphatidylinositol 4-kinase IIIalpha. Mol. Biol. Cell 19, 711-721 (2008)

22. Toth, J. T. et al. BRET-monitoring of the dynamic changes of inositol lipid pools in living cells reveals a PKC-dependent PtdIns4P increase upon EGF and M3 receptor activation. Biochim. Biophys. Acta 1861, 177-187 (2016).

23. Nakatsu, F. et al. PtdIns4P synthesis by PI4KIIIalpha at the plasma membrane and its impact on plasma membrane identity. J. Cell Biol. 199, 1003-1016 (2012).

24. Baskin, J. M. et al. The leukodystrophy protein FAM126A (hyccin) regulates PtdIns(4)P synthesis at the plasma membrane. Nat. Cell Biol. 18, 132-138 (2016).

25. Baird, D., Stefan, C., Audhya, A., Weys, S. \& Emr, S. D. Assembly of the PtdIns 4-kinase Stt4 complex at the plasma membrane requires Ypp1 and Efr3. J. Cell Biol. 183, 1061-1074 (2008).

26. $\mathrm{Wu}, \mathrm{X}$. et al. Structural insights into assembly and regulation of the plasma membrane phosphatidylinositol 4-kinase complex. Dev. Cell 28, 19-29 (2014).

27. Bojjireddy, N., Guzman-Hernandez, M. L., Reinhard, N. R., Jovic, M. \& Balla, T. EFR3s are palmitoylated plasma membrane proteins that control responsiveness to G-protein-coupled receptors. J. Cell Sci. 128, 118-128 (2015).

28. Lees, J. A. et al. Architecture of the human PI4KIIIalpha lipid kinase complex. Proc. Natl Acad. Sci. USA 114, 13720-13725 (2017).

29. Dornan, G. L. et al. Probing the architecture, dynamics, and inhibition of the PI4KIIIalpha/TTC7/FAM126 complex. J. Mol. Biol. 430, 3129-3142 (2018).

30. Lan, T. H., Liu, Q., Li, C., Wu, G. \& Lambert, N. A. Sensitive and high resolution localization and tracking of membrane proteins in live cells with BRET. Traffic 13, 1450-1456 (2012).

31. Chen, J. J., Fan, Y. \& Boehning, D. Regulation of dynamic protein S-acylation. Front Mol. Biosci. 8, 656440 (2021).

32. Gomez-Salinero, J. M., Pavia, P. G. \& Lara-Pezzi, E. CnAbetal shifts cardiac metabolism. Aging 11, 839-840 (2019).
33. Blanc, M. et al. SwissPalm: protein palmitoylation database. F1000Res 4, 261 (2015).

34. Forrester, M. T. et al. Site-specific analysis of protein S-acylation by resinassisted capture. J. Lipid Res. 52, 393-398 (2011).

35. Percher, A. et al. Mass-tag labeling reveals site-specific and endogenous levels of protein S-fatty acylation. Proc. Natl Acad. Sci. USA 113, 4302-4307 (2016)

36. Percher, A., Thinon, E. \& Hang, H. Mass-tag labeling using acyl-PEG exchange for the determination of endogenous protein S-fatty acylation. Curr. Protoc. Protein Sci. 89, 1417 11-14 1711 (2017).

37. Dallavilla, T. et al. Model-driven understanding of palmitoylation dynamics: regulated acylation of the endoplasmic reticulum chaperone calnexin. PLoS Comput. Biol. 12, e1004774 (2016).

38. Remsberg, J. R. et al. ABHD17 regulation of plasma membrane palmitoylation and N-Ras-dependent cancer growth. Nat. Chem. Biol. 17 , 856-864 (2021)

39. Lin, D. T. \& Conibear, E. ABHD17 proteins are novel protein depalmitoylases that regulate N-Ras palmitate turnover and subcellular localization. Elife $\mathbf{4}$ el1306 (2015).

40. Martin, B. R. \& Cravatt, B. F. Large-scale profiling of protein palmitoylation in mammalian cells. Nat. Methods 6, 135-138 (2009)

41. Zhang, M. M., Tsou, L. K., Charron, G., Raghavan, A. S. \& Hang, H. C. Tandem fluorescence imaging of dynamic S-acylation and protein turnover. Proc. Natl Acad. Sci. USA 107, 8627-8632 (2010).

42. St-Denis, N. et al. Phenotypic and interaction profiling of the human phosphatases identifies diverse mitotic regulators. Cell Rep. 17, 2488-2501 (2016).

43. Lambert, J. P. et al. Mapping differential interactomes by affinity purification coupled with data-independent mass spectrometry acquisition. Nat. Methods 10, 1239-1245 (2013).

44. Glatter, T., Wepf, A., Aebersold, R. \& Gstaiger, M. An integrated workflow for charting the human interaction proteome: insights into the PP2A system. Mol. Syst. Biol. 5, 237 (2009).

45. Sheftic, S. R., Page, R. \& Peti, W. Investigating the human Calcineurin Interaction Network using the piLxVP SLiM. Sci. Rep. 6, 38920 (2016).

46. Hauser, H. P., Bardroff, M., Pyrowolakis, G. \& Jentsch, S. A giant ubiquitinconjugating enzyme related to IAP apoptosis inhibitors. J. Cell Biol. 141 1415-1422 (1998)

47. Wei, Z. et al. Liprin-mediated large signaling complex organization revealed by the liprin-alpha/CASK and liprin-alpha/liprin-beta complex structures. Mol. Cell 43, 586-598 (2011).

48. Serfass, J. M. et al. Endophilin B2 facilitates endosome maturation in response to growth factor stimulation, autophagy induction, and influenza A virus infection. J. Biol. Chem. 292, 10097-10111 (2017).

49. Szymczak, A. L. et al. Correction of multi-gene deficiency in vivo using a single 'self-cleaving' 2 A peptide-based retroviral vector. Nat. Biotechnol. 22, 589-594 (2004).

50. Li, H., Rao, A. \& Hogan, P. G. Structural delineation of the calcineurin-NFAT interaction and its parallels to PP1 targeting interactions. J. Mol. Biol. 342, 1659-1674 (2004)

51. Gingras, A. C., Abe, K. T. \& Raught, B. Getting to know the neighborhood: using proximity-dependent biotinylation to characterize protein complexes and map organelles. Curr. Opin. Chem. Biol. 48, 44-54 (2018)

52. Roux, K. J., Kim, D. I., Raida, M. \& Burke, B. A promiscuous biotin ligase fusion protein identifies proximal and interacting proteins in mammalian cells. J. Cell Biol. 196, 801-810 (2012).

53. Oganesyan, I., Lento, C. \& Wilson, D. J. Contemporary hydrogen deuterium exchange mass spectrometry. Methods 144, 27-42 (2018).

54. Masson, G. R., Jenkins, M. L. \& Burke, J. E. An overview of hydrogen deuterium exchange mass spectrometry (HDX-MS) in drug discovery. Expert Opin. Drug Discov. 12, 981-994 (2017).

55. Hornbeck, P. V. et al. PhosphoSitePlus, 2014: mutations, PTMs and recalibrations. Nucleic Acids Res. 43, D512-D520 (2015)

56. Brombacher, E. et al. Rab1 guanine nucleotide exchange factor SidM is a major phosphatidylinositol 4-phosphate-binding effector protein of Legionella pneumophila. J. Biol. Chem. 284, 4846-4856 (2009).

57. Hendus-Altenburger, R. et al. Molecular basis for the binding and selective dephosphorylation of $\mathrm{Na}(+) / \mathrm{H}(+)$ exchanger 1 by calcineurin. Nat. Commun. 10, 3489 (2019).

58. Czirjak, G. \& Enyedi, P. Targeting of calcineurin to an NFAT-like docking site is required for the calcium-dependent activation of the background $\mathrm{K}+$ channel, TRESK. J. Biol. Chem. 281, 14677-14682 (2006).

59. Coghlan, V. M. et al. Association of protein kinase A and protein phosphatase 2B with a common anchoring protein. Science 267, 108-111 (1995).

60. Akimzhanov, A. M. \& Boehning, D. Rapid and transient palmitoylation of the tyrosine kinase Lck mediates Fas signaling. Proc. Natl Acad. Sci. USA 112, 11876-11880 (2015)

61. Dekker, F. J. et al. Small-molecule inhibition of APT1 affects Ras localization and signaling. Nat. Chem. Biol. 6, 449-456 (2010). 
62. Blom, N., Sicheritz-Ponten, T., Gupta, R., Gammeltoft, S. \& Brunak, S. Prediction of post-translational glycosylation and phosphorylation of proteins from the amino acid sequence. Proteomics 4, 1633-1649 (2004).

63. Zhang, H. et al. A subcellular map of the human kinome. Elife 10, e64943 (2021).

64. Liu, P. et al. PtdIns (3,4,5)P3-dependent activation of the mTORC2 kinase complex. Cancer Discov. 5, 1194-1209 (2015).

65. Ebner, M., Sinkovics, B., Szczygiel, M., Ribeiro, D. W. \& Yudushkin, I. Localization of mTORC2 activity inside cells. J. Cell Biol. 216, 343-353 (2017).

66. Schroder, W. A. et al. Human Sin1 contains Ras-binding and pleckstrin homology domains and suppresses Ras signalling. Cell. Signal. 19, 1279-1289 (2007).

67. Waterhouse, A. M., Procter, J. B., Martin, D. M., Clamp, M. \& Barton, G. J. Jalview Version 2-a multiple sequence alignment editor and analysis workbench. Bioinformatics 25, 1189-1191 (2009).

68. Barallon, R. et al. Recommendation of short tandem repeat profiling for authenticating human cell lines, stem cells, and tissues. In Vitro Cell Dev. Biol. Anim. 46, 727-732 (2010).

69. Almeida, J. L., Hill, C. R. \& Cole, K. D. Authentication of African green monkey cell lines using human short tandem repeat markers. BMC Biotechnol. 11, 102 (2011).

70. Schindelin, J. et al. Fiji: an open-source platform for biological-image analysis. Nat. Methods 9, 676-682 (2012).

71. Stauffer, W., Sheng, H. \& Lim, H. N. EzColocalization: an ImageJ plugin for visualizing and measuring colocalization in cells and organisms. Sci. Rep. 8, 15764 (2018).

72. Shteynberg, D. et al. iProphet: multi-level integrative analysis of shotgun proteomic data improves peptide and protein identification rates and error estimates. Mol. Cell. Proteom. 10, M111 007690 (2011).

73. Liu, G. et al. ProHits: integrated software for mass spectrometry-based interaction proteomics. Nat. Biotechnol. 28, 1015-1017 (2010).

74. Eng, J. K., Jahan, T. A. \& Hoopmann, M. R. Comet: an open-source MS/MS sequence database search tool. Proteomics 13, 22-24 (2013).

75. Keller, A., Nesvizhskii, A. I., Kolker, E. \& Aebersold, R. Empirical statistical model to estimate the accuracy of peptide identifications made by MS/MS and database search. Anal. Chem. 74, 5383-5392 (2002).

76. Nesvizhskii, A. I., Keller, A., Kolker, E. \& Aebersold, R. A statistical model for identifying proteins by tandem mass spectrometry. Anal. Chem. 75, 4646-4658 (2003).

77. Stariha, J. T. B., Hoffmann, R. M., Hamelin, D. J. \& Burke, J. E. Probing protein-membrane interactions and dynamics using hydrogen-deuterium exchange mass spectrometry (HDX-MS). Methods Mol. Biol. 2263, 465-485 (2021).

78. Dobbs, J. M., Jenkins, M. L. \& Burke, J. E. Escherichia coli and Sf9 contaminant databases to increase efficiency of tandem mass spectrometry peptide identification in structural mass spectrometry experiments. J. Am. Soc. Mass Spectrom. 31, 2202-2209 (2020).

79. Masson, G. R. et al. Recommendations for performing, interpreting and reporting hydrogen deuterium exchange mass spectrometry (HDX-MS) experiments. Nat. Methods 16, 595-602 (2019).

80. Perez-Riverol, Y. et al. The PRIDE database and related tools and resources in 2019: improving support for quantification data. Nucleic Acids Res. 47, D442-D450 (2019)

81. Cyert, M. \& Ulengin-Talkish, I. Palmitoylation targets the Calcineurin phosphatase to the Phosphatidylinositol 4-kinase complex at the plasma membrane. Mendeley Data. https://doi.org/10.17632/85v4dj4kgm.1 (2021).

\section{Acknowledgements}

We thank Callie Preast Wigington for critical reading of the manuscript, Rachel Bond for generating most of the CNA $\beta$ plasmids used in this study and for initiating experiments to identify CNA $\beta$-interacting proteins, Pin-Joe Ko for helpful discussions about quantitative image analysis, Jamin Hein for assistance in developing the phospho-specific FAM126A antibody, the Skotheim lab for cell lines, the De Camilli lab for plasmids and all members of the Cyert lab for their support and critical feedback. M.S.C., J.R. and I.U.T. are supported by grants from the National Institute of Health R01GM129236 and R35GM136243. J.E.B. acknowledges funding support from a Discovery grant from the Natural Science and Engineering Research Council of Canada (NSERC-2020-04241, J.E.B.), and the Michael Smith Foundation for Health Research (J.E.B., Scholar Award 17686). G.G. and T.B. are supported by the Intramural Research Program of the Eunice Kennedy Shriver National Institute of Child Health and Human Development of the NIH. A.C.G. is supported by a grant from the Canadian Institutes of Health Research (FDN 143301). P.V. is supported by the Hungarian National Research, Development, and Innovation Fund (NKFIK134357). E.C. acknowledges support from the Canadian Institutes of Health Research (PJT-162184).

\section{Author contributions}

I.U.-T. and M.S.C. jointly designed the study. I.U.-T. performed the majority of the experiments and analyzed data, supervised by M.S.C., with the exception of the following: M.A.H.P., M.L.J. and J.E.B. designed and executed HDX-MS experiments. J.R. carried out in vitro binding studies. A.Z.L.S. and E.C. designed and performed pulse-chase analyses of palmitate incorporation. N.St-D. and A.-C.G. designed and conducted AP-MS experiments. BRET-based analyses of PI4P dynamics were designed and executed by P.V. (effects of CN inhibitors) and by G.G. and T.B. (analyses of FAM126A mutants). I.U.-T. and M.S.C. wrote the manuscript with editorial input and approval from all authors.

\section{Competing interests}

The authors declare no competing interests.

\section{Additional information}

Supplementary information The online version contains supplementary material available at https://doi.org/10.1038/s41467-021-26326-4.

Correspondence and requests for materials should be addressed to Martha S. Cyert.

Peer review information Nature Communications thanks the anonymous reviewers for their contribution to the peer review of this work. Peer reviewer reports are available.

Reprints and permission information is available at http://www.nature.com/reprints

Publisher's note Springer Nature remains neutral with regard to jurisdictional claims in published maps and institutional affiliations.

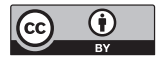

Open Access This article is licensed under a Creative Commons Attribution 4.0 International License, which permits use, sharing, adaptation, distribution and reproduction in any medium or format, as long as you give appropriate credit to the original author(s) and the source, provide a link to the Creative Commons license, and indicate if changes were made. The images or other third party material in this article are included in the article's Creative Commons license, unless indicated otherwise in a credit line to the material. If material is not included in the article's Creative Commons license and your intended use is not permitted by statutory regulation or exceeds the permitted use, you will need to obtain permission directly from the copyright holder. To view a copy of this license, visit http://creativecommons.org/ licenses/by/4.0/

\section{(C) The Author(s) 2021}

